\title{
Physical and psychosocial benefits of yoga in cancer patients and survivors, a systematic review and meta-analysis of randomized controlled trials
}

\author{
Laurien M Buffart ${ }^{\text {* }}$, Jannique GZ van Uffelen ${ }^{2,3}$, Ingrid I Riphagen ${ }^{4}$, Johannes Brug ${ }^{1}$, Willem van Mechelen ${ }^{5}$, \\ Wendy J Brown ${ }^{3}$ and Mai JM Chinapaw ${ }^{5}$
}

\begin{abstract}
Background: This study aimed to systematically review the evidence from randomized controlled trials (RCTs) and to conduct a meta-analysis of the effects of yoga on physical and psychosocial outcomes in cancer patients and survivors.

Methods: A systematic literature search in ten databases was conducted in November 2011. Studies were included if they had an RCT design, focused on cancer patients or survivors, included physical postures in the yoga program, compared yoga with a non-exercise or waitlist control group, and evaluated physical and/or psychosocial outcomes. Two researchers independently rated the quality of the included RCTs, and high quality was defined as $>50 \%$ of the total possible score. Effect sizes (Cohen's $d$ ) were calculated for outcomes studied in more than three studies among patients with breast cancer using means and standard deviations of post-test scores of the intervention and control groups.

Results: Sixteen publications of 13 RCTs met the inclusion criteria, of which one included patients with lymphomas and the others focused on patients with breast cancer. The median quality score was $67 \%$ (range: 22-89\%). The included studies evaluated 23 physical and 20 psychosocial outcomes. Of the outcomes studied in more than three studies among patients with breast cancer, we found large reductions in distress, anxiety, and depression ( $d=-0.69$ to -0.75$)$, moderate reductions in fatigue $(d=-0.51)$, moderate increases in general quality of life, emotional function and social function ( $d=0.33$ to 0.49$)$, and a small increase in functional well-being $(\mathrm{d}=0.31)$. Effects on physical function and sleep were small and not significant.
\end{abstract}

Conclusion: Yoga appeared to be a feasible intervention and beneficial effects on several physical and psychosocial symptoms were reported. In patients with breast cancer, effect size on functional well-being was small, and they were moderate to large for psychosocial outcomes.

Keywords: Yoga, Randomized controlled trial, Physical function, Psychosocial function, Quality of life, Cancer

\section{Background}

Cancer represents a major public health concern. In Western countries, approximately one in three persons will be directly affected by cancer before the age of 75 years, with breast cancer, melanoma, colorectal cancer and prostate cancer comprising the most common types $[1,2]$. Due to medical advances, survival rates have

\footnotetext{
* Correspondence: l.buffart@vumc.nl

'EMGO Institute for Health and Care Research, Department of Epidemiology and Biostatistics, VU University Medical Center, Van der Boechorststraat 7, Amsterdam 1081 BT, The Netherlands

Full list of author information is available at the end of the article
}

improved over the past decade. For example, currently, the 5-year survival rates across all cancers are approximately $56 \%$ for male and $62 \%$ for female patients in Australia [1] and 58\% and 64\%, respectively, in the Netherlands [2]. However, cancer and its treatment are often associated with prolonged adverse physical and psychosocial symptoms, including reduced physical function and fitness and increased risk of anxiety, depression, and fatigue $[3,4]$. This greatly impacts the patient's quality of life (QoL) $[5,6]$. Therefore, there is a need for effective methods to manage physical and psychosocial symptoms and to improve QoL of cancer patients and survivors.

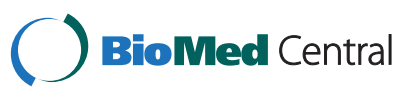


Psychosocial interventions such as counselling, support groups and cognitive behavioural therapies may help patients cope with cancer and the psychosocial problems associated with cancer and cancer treatment, but are less likely to help with common physical issues such as loss of strength and flexibility, weight gain, and reduced physical function [7]. Findings from previous reviews and meta-analyses suggest that aerobic and resistance exercise attenuate a range of the physical problems associated with cancer and cancer treatment $[3,4,6,8-16]$. The benefits of these types of exercise include not only improved physical function, but also reduced fatigue and improved QoL. Unfortunately, many cancer patients perceive various barriers to exercise [17-21]. The most common physical barriers are physical discomfort and feeling sick. Psychosocial barriers include having low mood, feelings of self-consciousness relating to appearance and body image, fatigue and fear for overdoing it [20,22,23]. Because of these barriers, approximately one out of three adult cancer patients turns to complementary and alternative medicine techniques, mindfulness, or yoga, to help manage their symptoms [24-26].

Yoga is a 'mind-body' exercise, a combination of physical poses with breathing and meditation [27]. Several studies in the non-cancer population reported positive effects of yoga on physical outcomes including perceptual and motor skills [28], cardiopulmonary function [29], fitness [30], muscle strength, flexibility, stiffness, and joint pain [31-33]. Furthermore, a recent review of 10 studies comparing the effects of yoga asanas (postures) with those of 'regular' exercise, indicated that yoga may be as effective as exercise for improving health outcomes such as blood glucose and lipids, fatigue, pain, and sleep in healthy people and in people with conditions such as diabetes and multiple sclerosis [34].

Previous reviews [35,36] and a meta-analysis [37] of intervention studies have reported that yoga is feasible for patients with cancer, with improved sleep, QoL, mood and levels of stress. The current study extends previous work by our exclusive focus on 1) randomised controlled trials (RCTs), the most rigorous intervention study design; 2) yoga interventions that included physical postures and were not part of a larger program such as MindfulnessBased Stress Reduction; and 3) a focus on both physical and psychosocial outcomes.

The aim of the present study is to conduct a systematic review and meta-analysis of the effects of yoga in cancer patients and survivors, focusing on both physical and psychosocial outcomes.

\section{Methods}

\section{Literature search}

IR, medical librarian, conducted the literature search in ten databases: AgeLine and AMED (Allied and Complementary
Medicine Database), British Nursing Index, CINAHL, CENTRAL (The Cochrane Central Register of Controlled Trials), EMBASE, PEDro, PsycINFO, PubMed and SPORTDiscus (earliest to November 2011). In order to identify all relevant papers, a search was conducted with both thesaurus terms and free terms for 'yoga' in combination with an extensive list of search terms to identify intervention studies. RCTs were identified using search terms for certain publication types (e.g. randomized controlled trial and controlled clinical trial in PubMed) in combination with a list of free text terms in title and abstracts that could be used to describe RCTs (e.g. randomi*ed, randomly, trial, groups). Detailed search profiles are available on request from IR. Additional articles were identified by manually checking the reference list of included papers.

\section{Study inclusion criteria}

Study inclusion criteria were: (i) design: RCT; (ii) population: adults with any cancer diagnosis either during or post treatment; (iii) intervention: yoga including physical postures (asanas); (iv) control group: non-exercise or wait-list; (v) outcome: physical and psychosocial outcomes. Only full-text articles written in English were included. Studies that included yoga as part of a larger intervention program (e.g., Mindfulness-Based Stress Reduction, meditation, or pranayama (breathing control) only) were excluded.

\section{Selection process and quality assessment}

Titles and abstracts of the references were reviewed to exclude articles out of scope (JvU). Full-text articles of potentially relevant records were assessed for eligibility by two independent reviewers (LB and JvU).

$\mathrm{LB}$ and $\mathrm{JvU}$ independently assessed the quality of the included papers using a Delphi list developed by Verhagen et al. [38], which consists of nine equally weighted quality criteria to assess different methodological aspects (see below). This list has previously been used for the evaluation of methodological quality in systematic reviews of exercise programs [39-41]. Criteria have a 'yes' (=1), 'no' (=0) or 'don't know' (=0) answer format. Disagreements between the reviewers were discussed and resolved, and in case of doubt, a third reviewer (MC) was consulted. Authors were contacted for additional information if it was not possible to score an item based on the information provided in the paper. Items scoring a "yes" contribute to the quality scores, ranging from 0 to 9 points. Where outcomes were assessed by self-report only, criterion 5 (blinding of the outcome assessor) was not applicable, and studies could obtain a maximum quality score of 8 points. A study was classified as a low quality study if the quality score was lower than $50 \%$ of the maximum possible score [41].

Criteria considered for quality assessment according to Verhagen et al. [38] 
1. Was a method of randomization performed?

2. Was the treatment allocation concealed?

3. Were the groups similar at baseline?

4. Were the eligibility criteria specified?

5. Was the outcome assessor blinded?

6. Was the yoga instructor blinded (i.e. unaware of the study aim)?

7. Was the participant blinded?

8. Were point estimates and measures of variability (between groups comparison) presented for the primary outcomes?

9. Did the analysis include an intention-to-treat analysis?

\section{Data extraction}

The following data were extracted by LB: (i) study population; (ii) type, intensity, frequency and duration of intervention, (iii) control group; (iv) outcome measures; and (v) effects on physical and/or psychosocial outcomes.

\section{Meta-analysis}

Effect sizes were calculated (standardized mean difference $d$ ) for all individual studies by subtracting the average post-test score of the control group $(\mathrm{Mc})$ from that of the yoga intervention group $(\mathrm{M} y)$ and dividing the result by the pooled standard deviations of the yoga intervention group and the control groups (SDyc) [42]. An effect size of 0.5 thus indicates that the mean of the experimental group is half a standard deviation larger than the mean of the control group. Effect sizes of 0.56 to 1.2 are large, while effect sizes of 0.33 to 0.55 are moderate and effect sizes of 0 to 0.32 are small [43].

For outcomes that were investigated in $>3$ studies, individual effect sizes were pooled in Comprehensive MetaAnalysis (CMA; version 2.2.046). Because only one study did not include patients with breast cancer [44], the metaanalyses was conducted on data from studies including patients with breast cancer only. As we expected considerable heterogeneity, we calculated pooled effect sizes with the random effects model. This model assumes that the included studies are drawn from 'populations' of studies that differ from each other systematically (heterogeneity). In this model, the prevalence resulting from the included studies not only differs because of the random error within studies (fixed effects model), but also because of true variation in prevalence from one study to the next. We first tested the heterogeneity under the fixed model using the statistics $\mathrm{I}^{2}$ and $\mathrm{Q}$. $\mathrm{I}^{2}$ describes the variance between studies as a proportion of the total variance. A value of $0 \%$ indicates no observed heterogeneity, and larger values show increasing heterogeneity, with $25 \%$ as low, $50 \%$ as moderate, and $75 \%$ as high heterogeneity [45]. When $P$ values of the $Q$ are above 0.05 , the total variance is due to variance within studies and not to variance between studies. We ran the analyses on all studies and with outliers excluded. Studies with extreme values of which the 95\% confidence interval had no overlap with the $95 \%$ confidence interval of the pooled estimate were considered as outliers.

\section{Results}

After removing duplicates, the literature searches yielded a total of 1909 unique records. For 171 potentially relevant records, we checked full text (Figure 1). The majority of the studies $(n=79)$ were excluded because they were not designed as a RCT. Of the records identified in the database search, 15 records met the inclusion criteria. We found one additional RCT [31] from the reference list of the review by Smith and Pukall [35]. Both Vadiraja et al. [46-48] and Raghavendra et al. [49,50] published more than one paper on the same RCT, each describing different outcome measures and/or subpopulations. Thus 16 papers [31,32,44,46-58] of 13 RCTs were included in this systematic review. Details of the populations, yoga interventions, and outcomes of the included studies are presented in Tables 1, 2 and 3.

\section{Quality assessment}

Results of the methodological quality assessment are presented in Table 4 . Median quality score was 67\% (range $22-89 \%$ ). All but one study [31] were of high quality. All included studies used randomization. In all but one [31] study treatment allocation was concealed, and groups were comparable at baseline, or dissimilarities at baseline were adequately adjusted for in the analyses. All studies adequately specified the eligibility criteria of the study population. The outcome assessor was blinded in five papers $[32,51,52,57,58]$, but this criterion was not applicable in the seven papers using self-reported outcomes only $[44,47,49,50,55]$. In five papers [51,52,55-57], the yoga instructor was blinded as he or she was unaware of the study aim. Participants were blinded in two papers [51,58]; Banerjee [51] informed us that their study was double blinded. In four papers, point estimates and $95 \%$ confidence intervals (CI) for between group differences were reported [47,50,54,58]. One paper [44] reported 95\% CI only, and three papers $[46,48,55]$ only presented effect sizes, without 95\% CI. In nine papers [32,47,48,50,52-55,58], data were analyzed on an intention-to-treat basis.

\section{Study population}

Details of the study populations are reported in Table 1. Twelve studies included patients with breast cancer and one study focused on patients with lymphomas [44]. Five studies in patients with breast cancer studies took place during cancer treatment: three studies (five papers [46-48,51,55]) during radiotherapy, one study [31] during hormone therapy, and one study (two papers, [49,50]) 


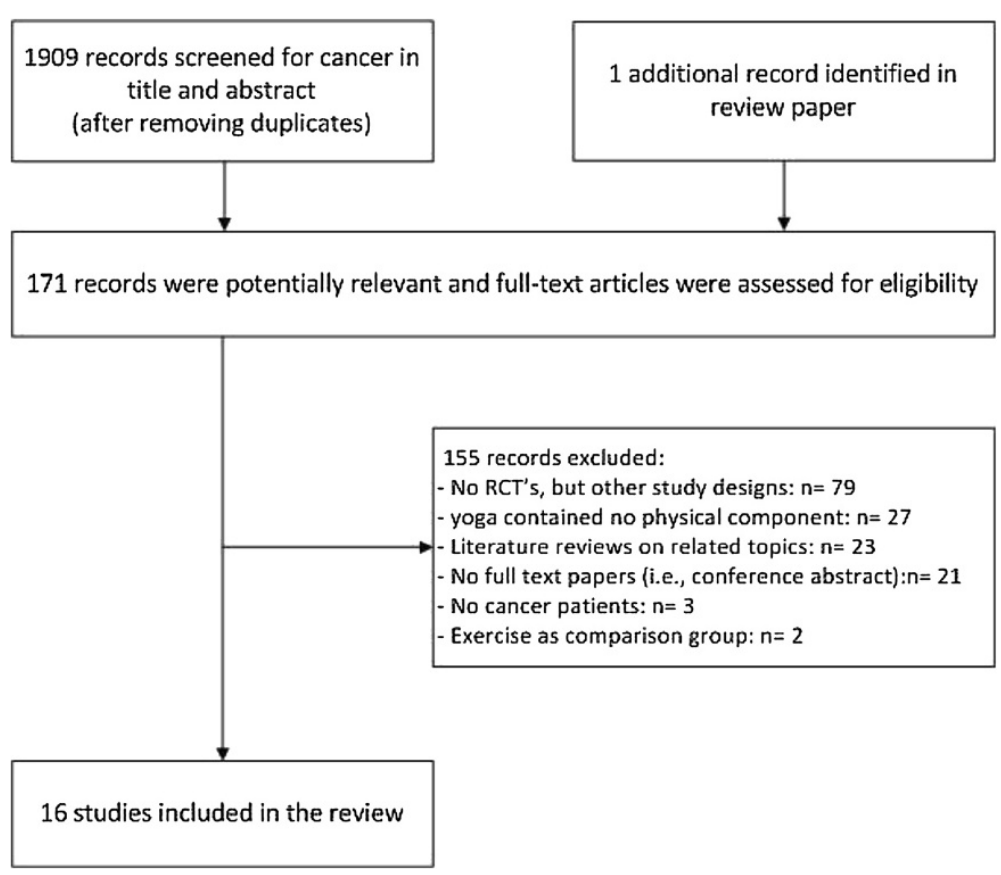

Figure 1 Flow chart.

during chemotherapy with or without additional radiotherapy. Five studies [32,52,56-58] focused on breast cancer survivors who had completed treatment, and two studies $[53,54]$ included patients and survivors both during and after treatment. The study in patients with lymphomas included patients during and after active treatment [44]. Sample sizes ranged from 18 to 128 patients, with seven studies including less than 50 patients, and only one study with more than 100 patients. Average age of the participants ranged from 44 to 63 years. One study did not report the age of the patients [50]. Eleven studies in patient with breast cancer included women only, one study [52] in mainly breast cancer patients (85\%) included 5\% men, and the study in lymphoma patients [44] included $39 \%$ men.

\section{Yoga program}

The content of the yoga programs is summarized in Table 2. All included a supervised yoga program with physical poses (yoga asanas), combined with breathing techniques (pranayama) and relaxation or meditation (savasana or dhanya).

All yoga classes were led by experienced yoga instructors. Median program duration was seven weeks with a range of six weeks to six months. In the study by Rao et al. [50], the program duration depended on the number of chemotherapy cycles, which ranged from four to eight. In this latter study, supervised sessions were conducted for 30 min before chemotherapy once every ten days. Furthermore, patients were provided with audiotapes of the exercises for home practice and asked to practice $1 \mathrm{~h}$ daily for 6 days/week during intervals between chemotherapy cycles [49]. In general, the number of classes per week ranged from one to three, and home practice was encouraged in nine studies, supported by audio or videotapes. Session duration ranged from 30 to $120 \mathrm{~min}$; three studies did not report the session duration $[31,44,50]$.

In nine studies [31,32,44,52-57] the yoga program was compared with a wait-list control group. In three studies [46-51], the control group received supportive therapy with education, counseling, or coping preparation. In one study, the control group received health education classes [58].

\section{Effects}

Tables 5 and 6 present an overview of the effects of yoga on physical and psychosocial outcomes, respectively (for details, see Table 3). Fourteen papers reported on both physical and psychosocial outcomes, and two papers reported on psychosocial outcomes only.

\section{Physical outcomes}

Twenty-three physical outcomes were examined in thirteen of the included papers (Table 5). In addition to selfreported physical function and functional well-being, outcomes included nine physical symptoms (e.g., pain, nausea, and dyspnoea), nine measures of physical activity and fitness, and three biological variables. However, except for physical function, functional well being, and pain, the outcomes were studied in only three studies or 
Table 1 Description of study populations in alphabetical order of first author

\begin{tabular}{lll}
\hline Author, year & Diagnosis; treatment & $\begin{array}{l}\text { number of participants ( } \mathbf{n}) ; \text { gender } \\
\text { (\%women); mean age (sd) and/or } \\
\text { range }\end{array}$ \\
\hline Banasik, 2011 [56] & Breast cancer, (>2 mo) post-treatment & $\begin{array}{l}n=18(9 Y, 9 C) \% \text { women: 100\% Age: } \\
62.9(7.1) \text { years }\end{array}$ \\
& & \\
& & \\
\hline Banerjee, 2007 [51] & Breast cancer, during radiotherapy & $\begin{array}{l}n=58(35 Y, 23 C) \% \text { women: 100\% } \\
\text { Age: 44 (1.3) years }\end{array}$
\end{tabular}

eligibility criteria

Inclusion women with stages II-IV breast cancer at least 2 months post-treatment.

Exclusion receiving Herceptin therapy, pregnant or lactating, had past or current history of

other neoplasm, active serious infection or immune deficiency; history of psychiatric disorders or alcohol or drug abuse; steroid therapy or physical condition preventing yoga.

Inclusion Recently operated breast cancer, age between 30 and 70 years, Zubrod's

performance status $0-2$ (ambulatory $>50 \%$ of the time), high school education, treatment plan of radiotherapy or both radiotherapy and chemotherapy, consent to participate in the study.

Exclusion Having any concurrent medical condition likely to interfere with the treatment; major psychiatric, neurological illness, or autoimmune disorders; cardiovascular illness; any known metastases. No exposure to other mutagens, smoking or alcohol for at least 3 months prior to pre-radiation blood donation.

\begin{tabular}{lll}
\hline Blank, 2003 [31] & $\begin{array}{l}\text { Breast cancer stage I-III receiving } \\
\text { antiestrogen or aromatase inhibitor } \\
\text { hormonal therapy }\end{array}$ & $\begin{array}{l}N=18 \text { (9Y, 9C) \% women: 100\% Age: } \\
48-69 \text { years }\end{array}$
\end{tabular}

Inclusion minimum of eight weeks post chemotherapy, estrogen receptor positive status, surgery for lumpectomy, modified mastectomy or full mastectomy (with/without reconstruction), a life expectancy greater than six months, adequate blood cell counts and kidney, liver, and cardiac function, physical and mental ability to attend all the Yoga training sessions.

Exclusion women on Herceptin therapy, current steroid therapy, or other known

immunomodulating medications, pregnancy or current lactation, a past or current history of another neoplasm, active serious infection or immune deficiency, documented alcohol or drug abuse, history of psychiatric disorders requiring use of psychotropic medication.

\begin{tabular}{lll}
\hline Bower, 2012 [58] & $\begin{array}{l}\text { Breast cancer state } 0-I I, \text { at least } \\
6 \text { months after adjuvant cancer therapy. }\end{array}$ & $n=31(16 \mathrm{Y}, 15 \mathrm{C}) \%$ women: $100 \%$ \\
& Ag.4 (5.7) years
\end{tabular}

Inclusion originally diagnosed with stage 0 to II breast cancer; completed local and/or adjuvant cancer therapy (with the exception of hormone therapy) at least 6 months previously; ages 40 to 65 years; postmenopausal; no other cancer in last 5 years; experiencing persistent cancer-related fatigue.

Exclusion chronic medical conditions or regular use of medications associated with fatigue; evidence that fatigue was driven primarily by a medical or psychiatric disorder other than cancer; evidence that fatigue was driven primarily by other noncancer-related factors; physical problems or conditions that could make yoga unsafe; a body mass index (BMI) $>31 \mathrm{~kg} / \mathrm{m} 2$.

\begin{tabular}{lll}
\hline Carson, 2009 [32] & Breast cancer; no current treatment (4.9 & $n=37$ (17Y, 20C) \% women: 100\% \\
& \pm 2.4 years since diagnose) & Age: $54.4(7.5)$ years
\end{tabular}

Inclusion Experiencing at least one hot flash per day on 4 or more days per week; no signs of active breast cancer; no current cytotoxic chemotherapy; diagnosed with breast cancer at stages $\mid \mathrm{A}-\| \mathrm{B} \geq 2$ years before; no hormone replacement therapy currently or within prior 3 months; stabilized on constant regime of menopausal symptom medications and supplements for at least 3 weeks; if taking antidepressants, stabilized at a fixed dose for at least 3 months.

Exclusion resided $\geq 70$ miles from research site; unavailable to attend the intervention on the day and at the time offered; currently engaged in intensive yoga practice ( $>3$ days/week); having received treatment for serious psychiatric disorders (e.g. schizophrenia) in the previous 6 months; not English speaking 
Table 1 Description of study populations in alphabetical order of first author (Continued)

\begin{tabular}{|c|c|c|c|}
\hline \multirow[t]{2}{*}{ Chandwani, 2010 [55] } & \multirow[t]{2}{*}{ Breast cancer, during radiotherapy } & \multirow[t]{2}{*}{$\begin{array}{l}N=61 \% \text { women: } 100 \% \text { Age: } 51.4 \\
\text { (8.0) range } 37-68 \text { years }\end{array}$} & $\begin{array}{l}\text { Inclusion Women with stage } 0 \text {-III breast cancer; } \geq 18 \text { years; able to read, write and speak } \\
\text { English; scheduled to undergo radiotherapy. }\end{array}$ \\
\hline & & & $\begin{array}{l}\text { Exclusion Patients who had any major psychiatric diagnosis or physical limitations that } \\
\text { would prohibit participation in the yoga program. }\end{array}$ \\
\hline \multirow[t]{2}{*}{ Cohen, 2004 [44] } & \multirow[t]{2}{*}{$\begin{array}{l}\text { Lymphoma (18\% Hodgkin), } 61,5 \% \text { active } \\
\text { treatment }\end{array}$} & \multirow[t]{2}{*}{$\mathrm{n}=39 \%$ women: $61.5 \%$ Age: 51 years } & $\begin{array}{l}\text { Inclusion Patients with lymphoma who were either receiving chemotherapy or had received } \\
\text { it within the past } 12 \text { months; } \geq 18 \text { years; able to read and speak English. }\end{array}$ \\
\hline & & & Exclusion Patients with major psychotic illnesses. \\
\hline Culos-Reed, 2006 [52] & $\begin{array}{l}\text { Breast cancer ( } 85 \%) \text {; no current } \\
\text { treatment (> } 3 \text { mo post-treatment) }\end{array}$ & $\begin{array}{l}n=38 \% \text { women: } 95 \% \text { Age: } 51.2 \\
\text { (10.3) years }\end{array}$ & $\begin{array}{l}\text { Inclusion Cancer survivors who were currently not undergoing active treatment; no } \\
\text { additional health concerns; } \geq 18 \text { years; minimum } 3 \text { months post-treatment. }\end{array}$ \\
\hline Danhauer, 2009 [53] & $\begin{array}{l}\text { Breast cancer; } 34 \% \text { actively undergoing } \\
\text { treatment }\end{array}$ & $\begin{array}{l}n=44 \% \text { women: } 100 \% \text { Age: } 55.8 \\
(9.9) \text { years }\end{array}$ & $\begin{array}{l}\text { Inclusion Women } \geq 18 \text { years; diagnosed with breast cancer; } 2 \text { to } 24 \text { months post-primary } \\
\text { treatment (surgery) following initial diagnosis and/or had a recurrence of breast cancer } \\
\text { within the past } 24 \text { months (regardless of treatment status); physically able to attend } \\
\text { restorative yoga; able to understand English; free of medical contraindications reported by } \\
\text { their physician. }\end{array}$ \\
\hline \multirow[t]{2}{*}{ Littman, 2011 [57] } & \multirow[t]{2}{*}{ Breast cancer; > 3 mo post-treatment } & \multirow[t]{2}{*}{$\begin{array}{l}n=63 \% \text { women: 100\% Age: } 60(7.9) \\
\text { years }\end{array}$} & $\begin{array}{l}\text { Inclusion Age between } 21 \text { and } 75 \text { years; completion of breast cancer treatment (stage 0-III) } \\
\text { at least } 3 \text { months prior, BMl } \geq 24 \mathrm{~kg} / \mathrm{m}^{2} \text { (or } \geq 23 \mathrm{~kg} / \mathrm{m}^{2} \text { if of Asian descent). }\end{array}$ \\
\hline & & & $\begin{array}{l}\text { Exclusion Myocardial infarction or stroke in the previous } 6 \text { months, diabetes, current yoga } \\
\text { practice, pregnancy or plans to become pregnant, factors that might lead to poor retention } \\
\text { and yoga practice. }\end{array}$ \\
\hline Moadel, 2007 [54] & Breast cancer; $48 \%$ medical treatment & $\begin{array}{l}n=128 \% \text { women: } 100 \% \text { Age: } 54.8 \\
\text { (9.9) range } 28-75 \text { years }\end{array}$ & $\begin{array}{l}\text { Inclusion Age } \geq 18 \text { years; new/recurrent breast cancer (stages I-III) diagnosis within previous } \\
5 \text { years; high performance status (Eastern Cooperative Oncology Group performance status } \\
\text { of }<3 \text { ); ability to speak English or Spanish; not actively practicing yoga. }\end{array}$ \\
\hline \multirow[t]{2}{*}{$\begin{array}{l}\text { Raghavendra, } 2007 \\
\text { [49] }\end{array}$} & \multirow[t]{2}{*}{ Breast cancer, during chemotherapy } & \multirow[t]{2}{*}{$\begin{array}{l}n=62 \% \text { women: } 100 \% \text { Age: } n=33 \\
<50 \text { yrs; } n=29>50 \text { yrs }\end{array}$} & $\begin{array}{l}\text { Inclusion Recently diagnosed with operable breast cancer; aged between } 30 \text { and } 70 \text { years; } \\
\text { Zubrod's performance status } 0-2 \text {; high school education; having a treatment plan with } \\
\text { surgery followed by adjuvant chemotherapy or by both adjuvant radiotherapy and } \\
\text { chemotherapy; consenting to participate in the study. }\end{array}$ \\
\hline & & & $\begin{array}{l}\text { Exclusion history of intestinal obstruction and any known sensitivity to any class of } \\
\text { antiemetics. }\end{array}$ \\
\hline \multirow[t]{2}{*}{ Rao, 2009 [50] } & \multirow[t]{2}{*}{$\begin{array}{l}\text { Breast cancer, during adjuvant } \\
\text { chemotherapy and radiotherapy }\end{array}$} & \multirow[t]{2}{*}{$\mathrm{n}=98 ; \%$ women: $100 \%$ Age: ? } & $\begin{array}{l}\text { Inclusion Recently diagnosed with operable breast cancer; aged between } 30 \text { and } 70 \text { years; } \\
\text { Zubrod's performance status } 0-2 \text {; high school education; having a treatment plan with } \\
\text { surgery followed by adjuvant radiotherapy and chemotherapy; consenting to participate in } \\
\text { the study. }\end{array}$ \\
\hline & & & $\begin{array}{l}\text { Exclusion Having a concurrent medical condition likely to interfere with the treatment; any } \\
\text { major psychiatric, neurological illness or autoimmune disorders; secondary malignancy. }\end{array}$ \\
\hline \multirow[t]{2}{*}{ Vadiraja, 2009 [46-48] } & \multirow[t]{2}{*}{$\begin{array}{l}\text { Breast cancer (stage } \| \text { and III), } \\
\text { during adjuvant }\end{array}$} & \multirow[t]{2}{*}{$\begin{array}{l}\mathrm{n}=88 ; \% \text { women: } 100 \% \text { Age: } 46 \text { (9.1) } \\
\text { yrs yoga; } 48.4(10.2) \text { yrs C. }\end{array}$} & $\begin{array}{l}\text { Inclusion Recently diagnosed with operable breast cancer; aged between } 30 \text { and } 70 \text { years; } \\
\text { Zubrod's performance status } 0-2 \text {; high school education; having a treatment plan with } \\
\text { surgery followed by adjuvant chemotherapy or by both adjuvant radiotherapy and } \\
\text { chemotherapy; consenting to participate in the study. }\end{array}$ \\
\hline & & & $\begin{array}{l}\text { Exclusion Having a concurrent medical condition likely to interfere with the treatment; any } \\
\text { major psychiatric, neurological illness or autoimmune disorders; any known metastases; } \\
\text { prescribed concurrent chemotherapy cycles during radiotherapy. }\end{array}$ \\
\hline
\end{tabular}


Table 2 Description of yoga programs, in alphabetical order of first author and attendance to yoga class

\section{Author, year $\quad$ Yoga program (Y); Duration and frequency (D); Home practice $(\mathrm{H})$ vs comparison $(\mathrm{C}) \quad$ Attendance}

Banasik 2011 [56] Y lyengar yoga given by expert lyenger instructors, with focus on training and accepting the Average 14 classes out of 16 (87.5\%), range 12 - 15.

physical form of the body without specific meditation component.

D 8 weeks, twice a week, 90 min per session

$\mathrm{H}$ -

C wait-list

Banerjee, 2007 [51] Y Meditative practice, slow stretching and loosening exercises, motivation and counseling,

yoga asanas, group awareness practices, pranayama, deep relaxation

yoga asanas, group awareness practices, prant
(yoga nidra) given by expert yoga trainers.

D 6-weeks; 90 min per session

H Patients were provided with audio and video tools to practice at home and were followed

up via telephone during weekends to ensure continuity of the practice.

C Supportive counseling and advised to take light exercise.

Blank, 2003 [31] Y Y lyengar Yoga, including seated meditation, active asana, restorative poses, savasana.

D 8 weeks, 2 times per week

H 1 home practice per week

C wait-list control

Bower, 2012 [58] Y Y lyengar yoga classes were taught by a certified Junior Intermediate lyengar yoga instructor and an assistant under the guidance of a senior teacher.

The mean number of yoga classes attended was 18.9 of 24 classes (78\%), and

D 12 weeks, twice a week, 90 min.

$\mathrm{H}$ -

C Health education classes were conducted for 120 min once a week for 12 weeks. Classes

were led by a PhD-level psychologist with clinical experience in the treatment of breast cancer survivors.

\begin{tabular}{|c|c|c|}
\hline \multirow[t]{4}{*}{ Carson, 2009 [32] } & $\begin{array}{l}\text { Y Yoga of Awareness given by certified yoga teacher: } 40 \text { min yoga poses, } 10 \text { min breathing } \\
\text { techniques, } 25 \text { min meditation, } 20 \text { min of study pertinent topics and } 25 \text { min group } \\
\text { discussion }\end{array}$ & \multirow[t]{4}{*}{ Average 6 classes out of $8(75 \%) .3$ women less than 4 classes $(3 / 17=17.6 \%)$} \\
\hline & D 8 weeks, once a week, 120 min & \\
\hline & $\begin{array}{l}\text { H Patients were encouraged to practice daily at home with aid of CD recordings and } \\
\text { illustrated hand books. }\end{array}$ & \\
\hline & C Wait-list control & \\
\hline \multirow[t]{4}{*}{ Chandwani, 2010 [55] } & $\begin{array}{l}\text { Y The multidimensional yoga module was given a trained yoga instructor: } 10 \text { warm-up } \\
\text { movements synchronized with breathing, } 25 \text { min maintenance in selected postures, } 10 \text { min } \\
\text { deep relaxation, } 5 \text { min pranayama, } 10 \text { min mediation. }\end{array}$ & \multirow{4}{*}{$\begin{array}{l}15(50 \%) \text { all } 12 \text { classes; } 8 \text { (28\%) attended } 11 \text { classes; } 1 \text { (3\%) attended } \\
10 \text { classes; } \\
1 \text { only } 2 \text { classes. One attended } 3 \text { classes, one 4, one 5, one } 7 \text { and one } 8 \\
\text { - classes. Average number of classes was } 10.2 \text { (85\%); SD: } 2.96 \text {; range } 2-12 \text {. } \\
\text { - Home practice: } \\
8 \text { (28\%) reported practicing }\end{array}$} \\
\hline & D 6 weeks, 2 times per week; 60 min per session & \\
\hline & $\begin{array}{l}\text { H Patients were encouraged to practice type full yoga once per day outside the classes, } \\
\text { supported by a } 60 \text {-min audio CD of the yoga program and a manual with photographs and } \\
\text { instructions. }\end{array}$ & \\
\hline & C Wait-list control & \\
\hline
\end{tabular}


Table 2 Description of yoga programs, in alphabetical order of first author and attendance to yoga class (Continued)

\begin{tabular}{|c|c|c|}
\hline \multirow[t]{4}{*}{ Cohen, 2004 [44] } & $\begin{array}{l}\text { Y Tibetan yoga sessions given by experienced instructor, divided into } 4 \text { aspects: controlled } \\
\text { breathing and visualization, mindfulness, and postures. }\end{array}$ & \multirow[t]{4}{*}{$32 \%$ all sessions; $26 \% 5$ or 6 sessions; $32 \% 2$ or 3 sessions; $10 \% 1$ session } \\
\hline & D 7 weekly sessions & \\
\hline & $\begin{array}{l}\text { H Patients were encouraged to practice the techniques at least once per day, supported by } \\
\text { audiotape that walked them though all of the techniques. }\end{array}$ & \\
\hline & C Wait-list control & \\
\hline \multirow[t]{4}{*}{ Culos-Reed, 2006 [52] } & $\begin{array}{l}\text { Y Classes were led by a certified yoga instructed and included } 10 \text { min gentle breathing; } \\
50 \text { min Yoga asanas; } 15 \text { min savasana. }\end{array}$ & \\
\hline & D 7 weeks, 75 min. & \\
\hline & $\mathbf{H}$ - & \\
\hline & C Wait-list control & \\
\hline \multirow[t]{4}{*}{ Danhauer, 2009 [53] } & $\begin{array}{l}\text { Y Restorative yoga classes were taught by a yoga instructor with cancer-specific yoga } \\
\text { training and combined yoga asanas, pranayama, savasana. }\end{array}$ & $\begin{array}{l}\text { Mean } 5.8 \text { (3.4) classes out of } 10(58 \%) 2(10 \%) \text { women 100\%; } 3 \text { (14\%) 0\% of } \\
\text { classes }\end{array}$ \\
\hline & D 10 weekly 75 -min classes & \\
\hline & $\mathbf{H}$ - & \\
\hline & C Wait-list control & \\
\hline \multirow[t]{4}{*}{ Littman, 2011 [57] } & $\begin{array}{l}\text { Y Viniyoga, a Hatha therapeutic type of yoga given by certified experienced yoga } \\
\text { instructors: 5-10 min centering exercises to promote relaxation and internal focus, } \\
50-60 \text { min of seated and standing poses, } 10-15 \text { min guided relaxation, breathing exercises } \\
\text { and meditation. }\end{array}$ & $\begin{array}{l}\text { Mean } 19.6 \text { (range 1-61; median 20.5) classes. Home practice: } 55.8 \text { times } \\
\text { (range } 2 \text { - 102; median 62). }\end{array}$ \\
\hline & D 6 months, 5 times per week including at least one 75 -min class & \\
\hline & $\begin{array}{l}\text { H patients were given a DVD, VD and booklets of four home practices lasting 20-30 min } \\
\text { each. }\end{array}$ & \\
\hline & C Wait-list control & \\
\hline \multirow[t]{4}{*}{ Moadel, 2007 [54] } & $\begin{array}{l}\text { Y Classes were given by a certified yoga instructor and included } 3 \text { yoga components: } \\
\text { physical stretches and poses, breathing exercises, and meditation. }\end{array}$ & $\begin{array}{l}\text { High adherence ( }>6 \text { classes): } n=33 \text { (; Low adherence ( } 1-6 \text { classes), } n=24 ; \text { No } \\
\text { adherence ( } 0 \text { classes), } n=27 \text {. Average attendance } 7 \text { out of } 12 \text { classes ( } 58 \%) \text {. }\end{array}$ \\
\hline & D 12 weekly 1.5 hrs classes (more allowed) & \\
\hline & $\begin{array}{l}\text { H Patients were asked to practice yoga at home daily and given an audiotape/compact disk } \\
\text { for guidance. }\end{array}$ & \\
\hline & C Wait-list control & \\
\hline \multirow[t]{4}{*}{ Raghavendra, 2007 [49] } & $\begin{array}{l}\text { Y Integrated yoga program administered by an instructor: asanas, breathing exercise, } \\
\text { pranayama, meditation and yogic relaxation techniques with imagery. }\end{array}$ & $?$ \\
\hline & $\begin{array}{l}\text { D } 30 \text { min before the start of the chemotherapy infusion (once in } 10 \text { days, number of cycles } \\
\text { 4-8). }\end{array}$ & \\
\hline & $\begin{array}{l}\text { H Patients were provided with audiotapes of these exercises for home practice and asked to } \\
\text { practice daily for } 1 \mathrm{~h} \text { for } 6 \text { days/week during intervals between chemotherapy cycles. }\end{array}$ & \\
\hline & C Supportive therapy and coping preparation & \\
\hline Rao, 2009 [50] & $\begin{array}{l}\text { Y Integrated yoga program administered by an instructor: asanas, breathing, pranayama, } \\
\text { mediation and yogic relaxation techniques with imagery. }\end{array}$ & $?$ \\
\hline
\end{tabular}

10 weekly 75-min classes

50-60 min of seated and standing poses, 10-15 min guided relaxation, breathing exercises

High adherence ( $>6$ classes): $\mathrm{n}=33$ (; Low adherence ( $1-6$ classes), $\mathrm{n}=24 ; \mathrm{No}$

12 weekly 1.5 hrs classes (more allowed)

fatients were asked to practice yoga at home daily and given an audiotape/compact disk

30 min before the start of the chemotherapy infusion (once in 10 days, number of cycles

Patients were provided with audiotapes of these exercises for home practice and asked to

practice daily for $1 \mathrm{~h}$ for 6 days/week during intervals between chemotherapy cycles.

mediation and yogic relaxation techniques with imagery. 
Table 2 Description of yoga programs, in alphabetical order of first author and attendance to yoga class (Continued)

\begin{tabular}{|c|c|c|}
\hline & $\begin{array}{l}\text { D Four sessions during pre- and post operative period, } 3 \text { in-person sessions per week for } \\
6 \text { weeks during radiotherapy. During chemotherapy, subjects underwent person sessions } \\
\text { during their hospital visits for chemotherapy administration (once in } 21 \text { days) and an } \\
\text { additional yoga session once in } 10 \text { days. }\end{array}$ & \\
\hline & H Patients were given booklets, audiotapes with instructions on practices for home practice. & \\
\hline & C Supportive therapy sessions & \\
\hline \multirow[t]{4}{*}{ Vadiraja, 2009 [46-48] } & $\begin{array}{l}\text { Y Integrated yoga program administered by an instructor: asanas, breathing, pranayama, } \\
\text { mediation and yogic relaxation techniques with imagery. }\end{array}$ & \multirow{4}{*}{$\begin{array}{l}29.7 \% \text { attended } 10-20 \% \text { supervised sessions, } 56.7 \% \text { attended } 20-25,13.7 \% \\
\text { attended }>25 \text { supervised sessions over a } 6 \text {-week period. Attend minimal 3x/ } \\
\text {-wk for } 6 \text { weeks } \rightarrow 18 \text { classes. }\end{array}$} \\
\hline & $\begin{array}{l}\text { D Minimum of } 3 \text { in-person sessions per week for } 6 \text { weeks during radio treatment; } 1 \text { hour per } \\
\text { session. In total between } 18-24 \text { yoga sessions. }\end{array}$ & \\
\hline & $\underline{\mathbf{H} \text { Patients were given booklets, audiotapes with instructions on practices for home practice. }}$ & \\
\hline & $\begin{array}{l}\text { C Supportive therapy with education. 15-min counseling sessions once every } 10 \text { days during } \\
6 \text { weeks ( } 3 \text { or } 4 \text { sessions in total). }\end{array}$ & \\
\hline
\end{tabular}


Table 3 Description of physical and psychosocial outcomes and between group differences (yoga vs control), in alphabetical order of first author

\begin{tabular}{|c|c|c|c|c|}
\hline Author, year & Physical outcomes & Between group difference & Psychosocial outcomes & Between group difference \\
\hline \multirow[t]{7}{*}{ Banasik, 2011 [56] } & FACT & & FACT & \\
\hline & - Physical well-being & N.S. & - emotional well-being & N.S. \\
\hline & - Functional well-being & N.S. & - social well-being & N.S. \\
\hline & Cortisol, morning & N.S. & Breast cancer concerns & N.S. \\
\hline & Cortison, noon & $P=0.004$ & Fatigue & $P=0.003$ \\
\hline & Cortisol, 5 p.m. & $P=0.004$ & & \\
\hline & Cortisol, 10 p.m. & N.S. & & \\
\hline \multirow[t]{3}{*}{ Banerjee, 2007 [51] } & DNA damage & $\begin{array}{l}14,5 \% \text { less DNA damage in } \\
\text { Yoga group; } p<0.001\end{array}$ & Anxiety (HADS-A) & $\begin{array}{l}48 \% \text { reduction in yoga group vs } 28 \% \\
\text { increase in controls; } p<0.001\end{array}$ \\
\hline & & & Depression (HADS-D) & $\begin{array}{l}57.5 \% \text { decrease in yoga vs } 24 \% \text { decrease } \\
\text { in controls; } p<0.001\end{array}$ \\
\hline & & & Perceived stress (PSS) & $\begin{array}{l}26.9 \% \text { reduction in yoga vs } 7 \% \text { increase in } \\
\text { controls; } \mathrm{p}<0.001\end{array}$ \\
\hline \multirow[t]{3}{*}{ Blank, 2003 [31] } & $\begin{array}{l}25 \% \text { had relieved joint aches and } \\
\text { shoulder stiffness }\end{array}$ & NA & $100 \%$ perceived direct stress reduction & NA \\
\hline & & & $\begin{array}{l}88 \% \text { felt more relaxed in daily life, more aware of body } \\
\text { posture, improved body image }\end{array}$ & NA \\
\hline & & & $63 \%$ had improved mood and less anxiety & NA \\
\hline \multirow[t]{5}{*}{ Bower, 2012 [58] } & $\begin{array}{l}\text { Lower extremity strength and } \\
\text { endurance (timed chair stands) }\end{array}$ & $1.31(-5.00 ; 2.38$, N.S. & Fatigue (FSI) & $-1.24(-0.04 ;-2.45), p<0.05$ \\
\hline & Flexibility (functional reach test) & $-2.00(5.76 ;-9.98)$, N.S. & Vigor & $4.80(1.86 ; 7.74), p<0.05$ \\
\hline & & & Depression (BDI) & $-5.80(-1.74 ;-9.86), p<0.05$ \\
\hline & & & Sleep quality (PSQI) & $0.20(2.78 ;-2.38)$, N.S. \\
\hline & & & Perceived stress (PSS) & $-1.77(1.71 ;-5.26)$, N.S. \\
\hline \multirow[t]{7}{*}{ Carson, 2009 [32] } & Hot flash frequency & $P=0.0017$ & Negative mood & $P=0.099$ \\
\hline & Hot flash severity & $P=0.0019$ & Relaxation & $P=0.543$ \\
\hline & Hot flash total & $P<0.0001$ & Vigor & $P=0.005$ \\
\hline & Joint pain & $P<0.0001$ & Acceptance & $P=0.058$ \\
\hline & Night sweats & N.S. & Symptom-related bother & $P<0.0001$ \\
\hline & & & Fatigue & $P=0.001$ \\
\hline & & & Sleep disturbance & $P=0.007$ \\
\hline \multirow[t]{4}{*}{ Chandwani, 2010 [55] } & SF-36 & & SF-36 & \\
\hline & - Physical component summary & $E S=0.44 ; P=0.04$ & - Mental component summary & N.S. \\
\hline & - Physical function & $E S=0.46 ; p=0.04$ & - Mental health & N.S. \\
\hline & - body pain & N.S. & - Role physical & N.S. \\
\hline
\end{tabular}


Table 3 Description of physical and psychosocial outcomes and between group differences (yoga vs control), in alphabetical order of first author (Continued)

\begin{tabular}{|c|c|c|c|c|}
\hline & & & - Role emotional & N.S. \\
\hline & & & - Social function & N.S. \\
\hline & & & - vitality & N.S. \\
\hline & & & - General HRQoL & $E S=0,47 ; p=0.005$ \\
\hline & & & Depression (CES-D) & N.S. \\
\hline & & & Anxiety (STAl) & N.S. \\
\hline & & & Distress (IES) & \\
\hline & & & - Intrusion & N.S. \\
\hline & & & - Avoidance & N.S. \\
\hline & & & Fatigue (BFI) & N.S. \\
\hline & & & Sleep (PSQI) & N.S. \\
\hline & & & Benefit finding (BFS) & N.S. \\
\hline \multirow[t]{12}{*}{ Cohen, 2004 [44] } & & & Distress (IES) & N.S. \\
\hline & & & Anxiety (STAI) & N.S. \\
\hline & & & Depression (CES-D) & N.S. \\
\hline & & & Sleep disturbances (PSQI) & \\
\hline & & & - Total score & $P=0.004$ \\
\hline & & & - Sleep quality & $P=0.02$ \\
\hline & & & - Sleep latency & $P=0.01$ \\
\hline & & & - Sleep duration & $P=0.03$ \\
\hline & & & - Sleep efficiency & N.S. \\
\hline & & & - Sleep medications & $P=0.02$ \\
\hline & & & - Daytime dysfunction & N.S. \\
\hline & & & Fatigue (BFI) & N.S. \\
\hline \multirow[t]{10}{*}{ Culos-Reed, 2006 [52] } & Physical activity (LSI) & N.S. & Mood (POMS) & \\
\hline & Weight & N.S. & - Total mood & $P<0.10$ \\
\hline & Systolic and diastolic blood pressure & N.S. & - Tension-anxiety & $P<0.10$ \\
\hline & Hand grip strength & N.S. & - Depression-dejection & $P<0.10$ \\
\hline & Distance walked & N.S. & - Confusion-bewilderment & $P<0.10$ \\
\hline & Perceived exertion & N.S. & - Vigor & N.S. \\
\hline & Flexibility (sit and reach) & N.S. & - Anger-hostility & N.S. \\
\hline & EORTC-QLQ-C30 & & Symptoms of stress (SOSI) & \\
\hline & - Physical function & N.S. & - Peripheral manifestations & N.S. \\
\hline & - pain & N.S. & - Cardiopulmonary symptoms & N.S. \\
\hline
\end{tabular}


Table 3 Description of physical and psychosocial outcomes and between group differences (yoga vs control), in alphabetical order of first author (Continued)

\begin{tabular}{|c|c|c|c|c|}
\hline & - nausea and vomiting & N.S. & - Symptoms of arousal & N.S. \\
\hline & - dyspnea & $P<0.05$ & - Upper respiratory symptoms & N.S. \\
\hline & - appetite & N.S. & - Central neurological symptoms & N.S. \\
\hline & - constipation & N.S. & - Gastrointestinal symptoms & $P<0.10$ \\
\hline & - diarrhea & $P<0.05$ & - Muscle tension & N.S. \\
\hline & & & - Habitual patterns & N.S. \\
\hline & & & - Depression & N.S. \\
\hline & & & - Anxiety/fear & N.S. \\
\hline & & & - Emotional irritability & $P<0.10$ \\
\hline & & & - Cognitive disorganization & $P<0.10$ \\
\hline & & & HRQoL (EORTC QLQ-C30) & \\
\hline & & & - global quality of life & $P<0.01$ \\
\hline & & & - emotional function & $P<0.05$ \\
\hline & & & - cognitive function & N.S. \\
\hline & & & - social function & N.S. \\
\hline & & & - role function & N.S. \\
\hline & & & - fatigue (POMS) & N.S. \\
\hline & & & - sleep disturbance & N.S. \\
\hline \multirow[t]{16}{*}{ Danhauer, 2009 [53] } & Physical function (SF-12) & N.S. & Mental health (SF-12) & $P=0.004$ \\
\hline & FACT & & Depression (CES-D) & $P=0.026$ \\
\hline & - Physical well-being & N.S. & Fatigue (FACT-fatigue) & N.S. \\
\hline & - Functional well-being & N.S. & Negative affect (PANAS-NA) & $P=0.014$ \\
\hline & & & Positive affect (PANAS-PA) & $P=0.01$ \\
\hline & & & FACT-General & $P=0.052$ \\
\hline & & & - Social well-being & N.S. \\
\hline & & & - Emotional well-being & $P=0.042$ \\
\hline & & & Spiritual well being (FACIT Sp) & \\
\hline & & & - peace/meaning & $P=0.0009$ \\
\hline & & & - role of faith & N.S. \\
\hline & & & Sleep disturbances (PSQI) & \\
\hline & & & - Total score & N.S. \\
\hline & & & - Sleep quality & N.S. \\
\hline & & & - Sleep latency & $P=0.078$ \\
\hline & & & - Sleep duration & N.S. \\
\hline
\end{tabular}


Table 3 Description of physical and psychosocial outcomes and between group differences (yoga vs control), in alphabetical order of first author (Continued)

\begin{tabular}{|c|c|c|c|c|}
\hline & & & - Sleep efficiency & N.S. \\
\hline & & & - Sleep medications & $P=0.10$ \\
\hline & & & - Daytime dysfunction & N.S. \\
\hline \multirow[t]{8}{*}{ Littman, 2011 [57] } & FACT & & Overall QoL (FACT-G) & N.S. \\
\hline & - Physical well-being & N.S. & Breast-cancer subscale & N.S. \\
\hline & - Functional well-being & N.S. & - Social well-being & N.S. \\
\hline & Physical Activity (MAQ) & N.S. & - Emotional well-being & N.S. \\
\hline & BMl & N.S. & - social/family well-being & N.S. \\
\hline & Waist circumference & $-3.1(-5.7 ;-0.4)$ & Fatigue (FACIT-F) & N.S. \\
\hline & Hip circumference & N.S. & & \\
\hline & weight & N.S. & & \\
\hline \multirow[t]{9}{*}{ Moadel, 2007 [54] } & FACT & & Overall QoL (FACT-G) & $P<0.01 \dagger$ \\
\hline & - Physical well-being & N.S. & - Social well-being & $E S=-0.22(-3.78$ to -0.36$) ; P=0.018$ \\
\hline & - Functional well-being & N.S. & - Emotional well-being & $P=0.018^{*} ; P<0.05 \dagger$ \\
\hline & & & Fatigue (FACT-fatigue) & N.S. \\
\hline & & & Spiritual well-being (FACIT Sp) & $P=0.009 \dagger$ \\
\hline & & & Distressed Mood (DMI) & $P<0.05 t$ \\
\hline & & & - Anxious/sad & $P=0.046 \dagger$ \\
\hline & & & - Irritability & $P=0.0275 t$ \\
\hline & & & - Confusion & N.S. \\
\hline \multirow[t]{6}{*}{ Raghavendra, 2007 [49] } & Nausea frequency & $P=0.01$ & Anxiety (STAI) & $P<0.001$ \\
\hline & Nausea severity & $P<0.01$ & Depression (DBI) & $P<0.001$ \\
\hline & Vomiting frequency & $P=0.06$ & Number of distressful symptoms & $P=0.002$ \\
\hline & Vomiting severity & $P=0.05$ & Severity of symptoms & $P<0.001$ \\
\hline & Total toxicity score & $P<0.001$ & Symptom distress & $P<0.001$ \\
\hline & & & Overall quality of life (FLIC) & $P<0.001$ \\
\hline \multirow[t]{3}{*}{ Rao, 2009 [50] } & & & State anxiety (STAI) & $E S=0.33 ; P<0.05(\mathrm{ITT})$ \\
\hline & & & Trait anxiety (STAl) & $\mathrm{ES}=0.24 ; \mathrm{NS}(\mathrm{ITT})$ \\
\hline & & & Symptom distress & $P=0.001$ \\
\hline \multirow[t]{4}{*}{ Vadiraja, 2009a [46] } & Cortisol level at 6 am & $E S=0.24 ; P<0.05$ & Anxiety (HADS-A) & $E S=0.31 ; P<0.001$ \\
\hline & Cortisol level at $9 \mathrm{am}$ & N.S. & Depression (HADS-D) & $E S=0.31 ; P<0.01$ \\
\hline & Cortisol level at $9 \mathrm{pm}$ & N.S. & perceived stress (PSS) & $E S=0.36 ; P<0.001$ \\
\hline & Mean pooled diurnal cortisol & $E S=0.27 ; P<0.05$ & & \\
\hline Vadiraja, 2009b [47] & EORTC QLQ-C30 & & Positive Affect (PANAS) & $E S=0.59 ; P=0.007$ \\
\hline
\end{tabular}


Table 3 Description of physical and psychosocial outcomes and between group differences (yoga vs control), in alphabetical order of first author (Continued)

\begin{tabular}{|c|c|c|c|c|}
\hline & - Physical function & $E S=0.16 ;$ N.S. & Negative Affect (PANAS) & $E S=0.84 ; P=0.001$ \\
\hline & & & \multicolumn{2}{|l|}{ HRQoL (EORTC QLQ-C30) } \\
\hline & & & - Role function & $E S=0.19 ;$ N.S. \\
\hline & & & - Emotional function & $E S=0.71 ; P=0.001$ \\
\hline & & & - Cognitive function & $E S=0.48 ; P=0.03$ \\
\hline & & & - Social function & $\mathrm{ES}=0.21 ; \mathrm{N} . \mathrm{S}$ \\
\hline \multirow[t]{9}{*}{ Vadiraja 2009c [48] } & Physical distress (RSCL) & $E S=0.33 ; p=0.02$ & Psychological distress (RCSL) & $E S=0.39 ; p<0.001$ \\
\hline & EORTC-QLQ-C30 & & EORTC QLQ-C30 & \\
\hline & - pain & $E S=0.14 ;$ N.S. & - fatigue & $\mathrm{ES}=0.33 ; \mathrm{N} . \mathrm{S}$ \\
\hline & - nausea and vomiting & $E S=0.05 ;$ N.S. & - insomnia & $\mathrm{ES}=0.47 ; \mathrm{N} . \mathrm{S}$ \\
\hline & - dyspnea & $E S=0.01 ;$ N.S. & & \\
\hline & - appetite loss & $E S=0.38 ;$ N.S. & & \\
\hline & - diarrhea & $E S=0.01 ;$ N.S. & & \\
\hline & - constipation & $E S=0.14 ;$ N.S. & & \\
\hline & Activity level & $E S=0.14 ;$ N.S. & & \\
\hline
\end{tabular}

BDI = Beck's Depression Inventory; BFI = Brief Fatigue Inventory; CES-D = Centers for Epidemiologic Studies-Depression; CT = chemotherapy; DMI = Distressed Mood Index; EORTC-QoL C30 = European Organization for

the Research and Treatment of Cancer-Quality of Life; ES = effect size; FACT-G = Functional Assessment of Cancer Therapy-General; FACIT = Functional Assessment of Chronic Illness Therapy; FLIC = Functional Living

Index for Cancer; FSI = Fatigue Symptom Inventory; HADS = Hospital Anxiety and Depression Scale; IES = Impact of Events Scale; ITT = Intention to treat; $L S I=$ Leisure Score Index; MAQ = Modifiable Activity

Questionnaire; NA = not assessed; N.S. = not significant; PANAS = Positive and Negative Effect Schedule; POMS = Profile of Mood states; PSQI = Pittsburgh Sleep Quality Index; PSS = perceived stress scale;

$\mathrm{RSCL}=$ Rotterdam Symptom Check List; SOSI = Symptoms of Stress Inventory; STAI = Spielberger's State Trait Anxiety Inventory. 
Table 4 Quality assessment sorted by study population and quality score

\begin{tabular}{|c|c|c|c|c|c|c|c|c|c|c|c|}
\hline First author, year & 1 & 2 & 3 & $4^{a}$ & 5 & 6 & 7 & 8 & 9 & score & $\%$ \\
\hline Banasik, 2011 [56] & Y & $Y$ & $\mathrm{Y}$ & $Y$ & SR & $Y^{C}$ & $\mathrm{~N}$ & $\mathrm{~N}$ & $N$ & 5 & $63 \%$ \\
\hline Banerjee, 2007 [51] & Y & $Y^{C}$ & Y & Y & Y & $Y^{C}$ & $Y^{C}$ & $\mathrm{~N}$ & $\mathrm{~N}$ & 7 & $78 \%$ \\
\hline Blank, 2003 [31] & Y & $?$ & $?$ & Y & $\mathrm{N}$ & $?$ & $\mathrm{~N}$ & $\mathrm{~N}$ & $\mathrm{~N}$ & 2 & $22 \%$ \\
\hline Bower, 2012 [58] & Y & Y & Y & Y & Y & $?$ & Y & Y & Y & 8 & $89 \%$ \\
\hline Carson, 2009 [32] & $\mathrm{Y}$ & $\mathrm{Y}$ & $\mathrm{Y}$ & $\mathrm{Y}$ & $Y$ & $\mathrm{~N}^{\mathrm{C}}$ & $\mathrm{N}$ & $N$ & $\mathrm{Y}$ & 6 & $67 \%$ \\
\hline Chandwani, 2010 [55] & $\mathrm{Y}$ & $Y^{C}$ & $Y$ & $\mathrm{Y}$ & SR & $Y^{C}$ & $\mathrm{~N}$ & $\mathrm{~N}, \mathrm{ES}$ no Cl & $\mathrm{Y}$ & 6 & $75 \%$ \\
\hline Cohen, 2004 [44] & Y & Y & Y & Y & $\mathrm{N}^{\mathrm{C}}(\mathrm{SR})$ & $\mathrm{N}^{\mathrm{C}}$ & $\mathrm{N}$ & $\mathrm{N}$, only $95 \% \mathrm{Cl}$ & $\mathrm{N}$ & 4 & $50 \%$ \\
\hline Culos-Reed, 2006 [52] & Y & Y & $\mathrm{Y}$ & $\mathrm{Y}$ & $Y^{C}$ & $Y^{C}$ & $\mathrm{~N}$ & $\mathrm{~N}$ & Y & 7 & $78 \%$ \\
\hline Danhauer, 2009 [53] & Y & $\mathrm{Y}$ & $\mathrm{Y}$ & $\mathrm{Y}$ & $N^{C}$ & $\mathrm{~N}^{\mathrm{C}}$ & $\mathrm{N}$ & $N$ & Y & 5 & $56 \%$ \\
\hline Littman, 2011 [57] & $\mathrm{Y}$ & $Y^{C}$ & $\mathrm{Y}$ & $\mathrm{Y}$ & $Y^{C}$ & $Y^{d}$ & $N$ & $\mathrm{Y}$ & $\mathrm{N}$ & 7 & $78 \%$ \\
\hline Moadel, 2007 [54] & $\mathrm{Y}$ & $Y^{C}$ & $Y^{b}$ & $\mathrm{Y}$ & $N^{C}$ & $N^{C}$ & $\mathrm{~N}$ & $\mathrm{Y}$ & Y & 6 & $67 \%$ \\
\hline Raghavendra, 2007 [49] & Y & Y & Y & Y & SR & $\mathrm{N}$ & $\mathrm{N}$ & $\mathrm{N}$ & $\mathrm{N}$ & 4 & $50 \%$ \\
\hline Rao, 2009 [50] & Y & Y & $Y$ & Y & SR & N & $\mathrm{N}$ & Y & Y & 6 & $67 \%$ \\
\hline Vadiraja, 2009a [46] & Y & Y & Y & Y & $?$ & $?$ & $\mathrm{~N}$ & $\mathrm{~N}, \mathrm{ES}$ no $\mathrm{Cl}$ & $\mathrm{N}$ & 4 & $50 \%$ \\
\hline Vadiraja, 2009b [47] & $Y$ & Y & $Y$ & $Y$ & SR & $?$ & $N$ & $Y$ & $Y$ & 6 & $75 \%$ \\
\hline Vadiraja, 2009c [48] & Y & $\mathrm{Y}$ & $\mathrm{Y}$ & $\mathrm{Y}$ & $S R$ & $?$ & $\mathrm{~N}$ & $\mathrm{~N}, \mathrm{ES}$ no $\mathrm{Cl}$ & $Y$ & 5 & $56 \%$ \\
\hline
\end{tabular}

$N A$ not applicable, $Y$ yes, $N$ no, ? unclear, ${ }^{a}$ If only exclusion criteria were reported, this was rated as 'unclear'; ${ }^{b}$ In the analyses, the baseline differences were included as covariates. ${ }^{\mathrm{C}}$ after contacting authors; ${ }^{\mathrm{d}}$ Yoga instructors were aware that the study aim was to determine the feasibility of conducting a yoga intervention in overweight and obese breast cancer survivors (not efficacy). SR self report, Cl Confidence interval, ES effect size.

less, thus we considered this evidence insufficient to draw conclusions on the effectiveness of yoga on these outcomes. After excluding an outlier [55], the pooled effect size of yoga on physical function in patients with breast cancer was small and insignificant $(d=0.17$; $95 \%$ $\mathrm{CI}=-0.06$ to 0.40 ), see Table 7 . Further, in patients with breast cancer, yoga resulted in a small but significant increase in functional well-being $(d=0.31 ; 95 \% \mathrm{CI}=0.04$ to 0.58). Pain was evaluated in four studies, of which standard deviations to calculate effect sizes were not available in two studies [32,44]. The average effect size of the other two studies among patients with breast cancer $[48,55]$ was large $(d=-0.64 ; 95 \% \mathrm{CI}=-0.98$ to -0.31$)$.

\section{Psychosocial outcomes}

Twenty psychosocial outcomes were examined in the fifteen included papers (Table 6). The effects of yoga on distress, anxiety, depression, fatigue, sleep, general QoL, emotional function and social function were evaluated in three or more studies. After excluding outliers, yoga resulted in significant large reductions in distress $(d=-0.75 ; 95 \% \mathrm{CI}=-1.09$ to -0.42$)$, anxiety $(d=-0.77$; $95 \% \mathrm{CI}=-1.08$ to -0.46$)$, and depression $(d=-0.69 ; 95 \%$ $\mathrm{CI}=-1.02$ to -0.37$)$, moderate reductions in fatigue $(d=-0.51 ; 95 \% \mathrm{CI}=-0.79$ to -0.22$)$, and moderate increases in general HRQoL $(d=0.37 ; 95 \% \mathrm{CI}=0.11$ to $0.62)$, emotional function $(d=0.49 ; 95 \% \mathrm{CI}=0.16$ to 0.81$)$, and social function $(d=0.33 ; 95 \% \mathrm{CI}=0.12$ to 0.54$)$ in breast cancer patients, see Table 7. Effects on sleep disturbances were small and insignificant $(d=-0.26 ; 95 \%$ $\mathrm{CI}=-0.53$ to 0.02 ). In patients with lymphoma, however, Cohen et al. [44] found a significant reduction in sleep disturbances $(d=-1.00 ; 95 \% \mathrm{CI}=-3.8$ to -0.8$)$. Although some studies found beneficial effects on other psychosocial outcomes, including positive and negative effect, mood, spirituality and relaxation (Table 6), these were studied in less than three studies. Therefore, this evidence was considered to be insufficient.

\section{Dropout and attendance}

Dropout from the studies, defined as the number of randomized participants without post-intervention measurement ranged from 0 to $38 \%$. Attendance at the yoga classes was reported in nine studies [32,44,47,53-57], and varied between 58 and $88 \%$ (Table 2). Vadiraja et al. [47] reported that the level of adherence did not influence results on QoL, positive and negative affect [47]. Four other studies reported on the influence of intervention adherence on outcomes. Danhauer et al. [53] reported that better intervention adherence was associated with higher self-reported physical function and QoL. In contrast, Moadel et al. [54] found similar improvements in QoL among participants with low and high class attendance, but found a positive association between intervention attendance and improved mood. Carson et al. [32] also showed that greater mean yoga practice time was associated with less fatigue, less symptom bother, and more acceptance at post-treatment, and tended to be associated 
Table 5 Summary of the effects of yoga compared to control on physical outcomes

\begin{tabular}{|c|c|c|c|c|c|c|c|c|c|c|c|c|c|c|c|c|}
\hline PHYSICAL & 1 & 2 & 3 & 4 & 5 & 6 & 7 & 8 & 9 & 10 & 11 & 12 & 13 & 14 & 15 & 16 \\
\hline Reference & {$[56]$} & {$[51]$} & [31] & {$[58]$} & {$[32]$} & {$[55]$} & [44] & {$[52]$} & {$[53]$} & [57] & {$[54]$} & [49] & {$[50]$} & {$[46]$} & {$[47]$} & {$[48]$} \\
\hline Year & 2011 & 2007 & 2003 & 2011 & 2009 & 2010 & 2004 & 2006 & 2009 & 2011 & 2007 & 2007 & 2009 & $2009 a$ & $2009 b$ & $2009 c$ \\
\hline Sample size & 18 & 58 & 18 & 31 & 37 & 61 & 39 & 38 & 44 & 63 & 128 & 62 & 98 & 88 & 88 & 88 \\
\hline Treatment & AT & RT & $\mathrm{HT}$ & AT & AT & RT & Mix & AT & Mix & AT & $\operatorname{mix}$ & CT & $C T+R T$ & RT & RT & $\mathrm{RT}$ \\
\hline Quality & high & high & low & high & high & high & high & high & high & high & high & high & high & high & high & high \\
\hline \multicolumn{17}{|l|}{ Physical function } \\
\hline Physical function & N.S. & & & & & $\uparrow$ & & N.S. & N.S. & N.S. & N.S. & & & & N.S. & \\
\hline Functional well being & N.S. & & & & & & & & N.S. & N.S. & N.S. & & & & & \\
\hline \multicolumn{17}{|l|}{ Physical symptoms } \\
\hline Pain & & & & & $\downarrow$ & N.S. & & N.S. & & & & & & & & N.S. \\
\hline Nausea vomiting & & & & & & & & N.S. & & & & $\downarrow$ & & & & N.S. \\
\hline Toxicity & & & & & & & & & & & & $\downarrow$ & & & & \\
\hline Diarrhoea & & & & & & & & $\downarrow$ & & & & & & & & N.S. \\
\hline Constipation & & & & & & & & N.S. & & & & & & & & N.S. \\
\hline Appetite & & & & & & & & N.S. & & & & & & & & N.S. \\
\hline Dyspnea & & & & & & & & $\downarrow$ & & & & & & & & N.S \\
\hline Hot flashes & & & & & $\downarrow$ & & & & & & & & & & & \\
\hline Night sweats & & & & & N.S. & & & & & & & & & & & \\
\hline \multicolumn{17}{|l|}{ Activity/fitness } \\
\hline Physical Activity & & & & & & & & N.S. & & N.S. & & & & & & N.S. \\
\hline Weight & & & & & & & & N.S. & & N.S. & & & & & & \\
\hline Body mass index & & & & & & & & & & N.S. & & & & & & \\
\hline Waist circumference & & & & & & & & & & $\downarrow$ & & & & & & \\
\hline Hip circumference & & & & & & & & & & N.S. & & & & & & \\
\hline Flexibility & & & & N.S. & & & & N.S. & & & & & & & & \\
\hline Strength & & & & N.S. & & & & N.S. & & & & & & & & \\
\hline Fitness/distance walked & & & & & & & & N.S. & & & & & & & & \\
\hline Perceived exertion & & & & & & & & N.S. & & & & & & & & \\
\hline \multicolumn{17}{|l|}{ Biological Variables } \\
\hline DNA Damage & & $\downarrow$ & & & & & & & & & & & & & & \\
\hline Cortisol & $\downarrow$ & & & & & & & & & & & & & $\downarrow$ & & \\
\hline Blood pressure & & & & & & & & N.S. & & & & & & & & \\
\hline
\end{tabular}

with less sleep disturbances. Littman et al. [57] reported that generally, the benefits were greater among women who attended more facility-based classes, but results were not entirely consistent.

\section{Safety}

Five studies evaluated adverse events and provided this information in the manuscripts $[47,50,53,57,58]$. Four studies reported that there were no adverse events and one study [58] reported one adverse event of a participant with a history of back problems, who experienced a back spasm in yoga class. After evaluation by her physician, she was able to return to class and complete the intervention.

\section{Discussion}

This review and meta-analysis described and evaluated sixteen papers examining yoga as an intervention to manage physical and psychosocial symptoms in cancer patients and survivors. In contrast to previous reviews $[35,36]$ and meta-analysis [37] we only included studies focusing on yoga interventions with physical postures, and evaluating the effectiveness on physical and/or psychosocial outcomes. Yoga appeared to be a feasible intervention, and beneficial effects on several physical and psychosocial symptoms were reported, with a small effect on functional well-being and moderate to large effects on various psychosocial outcomes. 
Table 6 Summary of the effects of yoga compared to control on psychosocial outcomes

\begin{tabular}{|c|c|c|c|c|c|c|c|c|c|c|c|c|c|c|c|c|}
\hline PSYCHOSOCIAL & 1 & 2 & 3 & 4 & 5 & 6 & 7 & 8 & 9 & 10 & 11 & 12 & 13 & 14 & 15 & 16 \\
\hline Reference & {$[56]$} & [51] & [31] & {$[58]$} & [32] & {$[55]$} & [44] & [52] & [53] & [57] & [54] & [49] & [50] & [46] & [47] & [48] \\
\hline Year & 2011 & 2007 & 2003 & 2011 & 2009 & 2010 & 2004 & 2006 & 2009 & 2011 & 2007 & 2007 & 2009 & $2009 a$ & $2009 b$ & $2009 c$ \\
\hline Sample size & 18 & 58 & 18 & 31 & 37 & 61 & 39 & 38 & 44 & 63 & 128 & 62 & 98 & 88 & 88 & 88 \\
\hline Treatment & AT & RT & $\mathrm{HT}$ & AT & AT & RT & Mix & AT & Mix & AT & Mix & $\mathrm{CT}$ & $\mathrm{CT}+\mathrm{RT}$ & RT & RT & RT \\
\hline Quality & high & high & low & high & high & high & High & high & high & high & high & high & high & high & high & high \\
\hline Distress & & $\downarrow$ & $\downarrow$ & N.S. & $\downarrow$ & N.S. & N.S. & & & & & $\downarrow$ & $\downarrow$ & $\downarrow$ & & $\downarrow$ \\
\hline Anxiety & & $\downarrow$ & $\downarrow$ & & & N.S. & N.S. & & & & $\downarrow$ & $\downarrow$ & $\downarrow$ & $\downarrow$ & & \\
\hline Depression & & $\downarrow$ & & $\downarrow$ & & N.S. & N.S. & & $\downarrow$ & & & $\downarrow$ & & $\downarrow$ & & N.S. \\
\hline Fatigue & $\downarrow$ & & & $\downarrow$ & $\downarrow$ & N.S. & N.S. & & N.S. & N.S. & N.S. & & & & & \\
\hline Sleep disturbance & & & & N.S. & $\downarrow$ & N.S. & $\downarrow$ & & N.S. & & & & & & & $\downarrow$ \\
\hline General HRQoL & $\uparrow$ & & & & & $\uparrow$ & & $\uparrow$ & $\uparrow$ & N.S. & $\uparrow$ & $\uparrow$ & & & & \\
\hline Emotional function & N.S. & & & & & N.S. & & & $\uparrow$ & N.S. & $\uparrow$ & & & & $\uparrow$ & \\
\hline Social function & N.S. & & & & & N.S. & & N.S. & N.S. & N.S. & $\uparrow$ & & & & N.S. & \\
\hline Role function & & & & & & N.S. & & & & & & & & & N.S. & \\
\hline Cognitive function & & & & & & & & & & & & & & & $\uparrow$ & \\
\hline Positive affect & & & & & & & & & $\uparrow$ & & & & & & $\uparrow$ & \\
\hline Negative affect & & & & & & & & & $\uparrow$ & & & & & & $\uparrow$ & \\
\hline Vigor & & & & $\uparrow$ & $\uparrow$ & & & & & & & & & & & \\
\hline Mood & & & $\uparrow$ & & $\uparrow$ & & & & & & & & & & & \\
\hline Anger-hostility & & & & & & & & & & & $\downarrow$ & & & & & \\
\hline Spirituality & & & & & & & & & $\uparrow$ & & $\uparrow$ & & & & & \\
\hline Relaxation & & & $\uparrow$ & & $\uparrow$ & & & & & & & & & & & \\
\hline Confusion & & & & & & & & & & & N.S. & & & & & \\
\hline Mental Health & & & & & & N.S. & & & $\uparrow$ & & & & & & & \\
\hline Acceptance & & & & & $\uparrow$ & & & & & & & & & & & \\
\hline
\end{tabular}

\section{Physical outcomes}

Due to the limited number of studies per physical outcome, evidence for physical effects of yoga was generally insufficient to draw firm conclusions. The effects of yoga on physical function and functional well-being were small. This may be related to the short intervention duration; only two studies lasted 12 weeks or longer [54,57], all others were shorter, ranging from 6 to 10 weeks (median $=7$ ). To improve physical function and fitness, longer intervention duration may be required. The lack of significant improvements in physical function and fitness may also be related to the relatively low intensity of certain types of yoga [52,59]. Nevertheless, in healthy older adults, a 6-month yoga intervention resulted in improved physical outcomes such as timed 1-leg stand, flexibility, and energy [60]. These beneficial effects may be related to the lower baseline cardiorespiratory fitness of older adults compared with younger patients, and the longer intervention duration in that specific study. Significant improvements in treadmill time and estimated peak oxygen uptake as a result of yoga have also been shown in a small group of patients with chronic heart failure [61]. A systematic review of studies comparing yoga with other forms of exercise concluded that in both healthy people and in patients with chronic diseases, yoga may be as effective or better than other forms of exercise at improving a variety of health-related outcome measures, including physical outcomes such as muscle strength and flexibility [34]. One study with healthy sedentary elderly people has reported that peak oxygen uptake increased by $11 \%$ after yoga, compared with $24 \%$ after aerobic training [62]. Although patients perceived that they had improved fitness after 12 weeks of yoga [63], future empirical evidence should indicate whether yoga is as beneficial as endurance or strength exercise in improving physical fitness in (physically inactive) cancer patients.

\section{Psychosocial outcomes}

This review found that yoga has large beneficial effects on distress, anxiety and depression, moderate beneficial effects on fatigue, general HRQoL, emotional function and social function, and a small and insignificant effect on sleep. There was insufficient evidence for effects on psychosocial outcomes that were studied less frequently 
Table 7 Pooled effects of yoga on physical and psychosocial outcomes in patients with breast cancer

\begin{tabular}{|c|c|c|c|c|c|c|c|c|}
\hline Outcome & \# studies & Pooled effect & & & & Test of heterogeneity & & \\
\hline Physical outcomes & & $d$ & $95 \% \mathrm{Cl}$ & $\mathbf{Z}$ & $\mathbf{P}$ & $I^{2}$ & $\mathbf{Q}$ & $\mathbf{P}$ \\
\hline \multirow[t]{2}{*}{ Physical function } & 6 & 0.60 & -0.05 to 1.25 & 1.81 & 0.07 & 87.51 & 40.03 & $<0.0001$ \\
\hline & $5^{a}$ & 0.17 & -0.06 to 0.40 & 1.48 & 0.14 & 0.00 & 1.20 & 0.88 \\
\hline Functional well-being & 4 & 0.31 & 0.04 to 0.58 & 2.24 & 0.03 & 0.00 & 1.25 & 0.74 \\
\hline Psychosocial outcomes & & $d$ & $95 \% \mathrm{Cl}$ & $z$ & $\mathbf{P}$ & $1^{2}$ & Q & $\mathbf{P}$ \\
\hline \multirow[t]{2}{*}{ Distress } & 7 & -0.95 & -1.49 to -0.49 & -4.04 & $<0.001$ & 80.79 & 31.24 & $<0.001$ \\
\hline & $6^{b}$ & -0.75 & -1.09 to -0.42 & -4.39 & $<0.001$ & 59.59 & 12.37 & 0.03 \\
\hline \multirow[t]{2}{*}{ Anxiety } & 7 & -1.25 & -1.93 to -0.56 & -3.64 & $<0.001$ & 91.45 & 70.20 & $<0.001$ \\
\hline & $6^{b}$ & -0.77 & -1.08 to -0.46 & -4.86 & $<0.001$ & 58.42 & 12.03 & 0.03 \\
\hline \multirow[t]{2}{*}{ Depression } & 7 & -1.47 & -2.42 to -0.53 & -3.05 & 0.002 & 93.29 & 89.46 & $<0.001$ \\
\hline & $6^{b}$ & -0.69 & -1.02 to -0.37 & -4.21 & $<0.001$ & 42.15 & 8.64 & 0.12 \\
\hline Fatigue & 7 & -0.51 & -0.79 to -0.22 & -3.46 & 0.001 & 43.52 & 10.62 & 0.10 \\
\hline Sleep disturbance & 4 & -0.26 & -0.53 to 0.02 & -1.82 & 0.07 & 0.00 & 1.25 & 0.74 \\
\hline \multirow[t]{3}{*}{ General HRQoL } & 7 & 0.88 & 0.25 to 1.50 & 2.75 & 0.006 & 86.49 & 44.41 & $<0.001$ \\
\hline & $6^{a}$ & 0.61 & 0.16 to 1.06 & 2.50 & 0.008 & 69.79 & 16.55 & 0.005 \\
\hline & $5^{a, c}$ & 0.37 & 0.11 to 0.62 & 2.85 & 0.004 & 0.00 & 3.40 & 0.49 \\
\hline Emotional function & 5 & 0.49 & 0.16 to 0.81 & 2.93 & 0.003 & 26.58 & 5.45 & 0.24 \\
\hline Social function & 6 & 0.33 & 0.12 to 0.54 & 3.12 & 0.002 & 0.00 & 1.94 & 0.86 \\
\hline
\end{tabular}

${ }^{a}$ Excluding outlier Chandwani et al. 2010 [55]; ${ }^{\text {b }}$ Excluding outlier Banerjee et al. [51]. ${ }^{c}$ Excluding outlier Raghavendra et al. 2007 [49].

including cognitive function, vigor, anger-hostility, spirituality, relaxation and mental health. More studies evaluating the effects of yoga on these outcomes are needed before we can draw firm conclusions.

The finding that yoga improves QoL, and reduces distress and depression concurs with findings from previous reviews and meta-analysis of yoga interventions for cancer patients and survivors [35,37]. In contrast, the current meta-analysis could not confirm previous findings on reductions in sleep disturbances in patients with breast cancer. Fatigue is among the most frequently occurring and debilitating complaints associated with cancer and cancer treatments $[5,64]$. Therefore, it is important to find effective strategies to reduce fatigue in cancer patients. In contrast to the meta-analysis of Lin et al. [37], we found a moderate significant effect size on fatigue. This is in line with a recent study of Bower et al. [58] who showed beneficial effects after 12 weeks of yoga classes on persistent fatigue in breast cancer survivors. In addition, patients themselves also perceived improvements in QoL, fatigue, stress, anxiety and depression [63]. The moderate-tolarge effect sizes on these psychosocial outcomes seem larger than the small to moderate effect sizes of exercise $[12,16,65-67]$ or psychosocial interventions $[12,66,68]$. However, our results have to be interpreted with caution due to small sample sizes in most studies. Furthermore effect sizes may also be influenced by patient selection, i.e. including also non-fatigued or non-depressed patients. Therefore, future studies should obtain insight in the most effective interventions to improve psychosocial outcomes. The current meta-analysis showed that yoga may be such an intervention.

\section{Methodological quality of studies}

This review included a quality rating, and only one paper was of low quality. A major concern regarding the methodological quality of most included studies was that not all participants completed the yoga program and data were not analysed on an intention-to-treat basis. This may have introduced bias, overestimating the benefits of yoga. Only half of the studies reported class attendance, of which four studies indicated that intervention adherence was positively associated with some outcomes [32,53,54,57]. Whether adherence to the yoga sessions was affected by cancer-related symptoms or side-effects of cancer treatment was not reported.

Most studies separately reported the descriptive results of the outcomes for the yoga and control groups, and presented only p-values for the group differences. Many studies however, did not report effect sizes or other point estimates of the between-group differences, and their confidence intervals. Therefore we calculated standardized mean differences using means and standard deviations of the post-test values.

Furthermore, as with all exercise interventions, blinding was difficult. Because the control group usually consisted of either wait-list or usual care, participants were not blinded to the intervention, possibly introducing bias. 


\section{Strengths and limitations}

The extensive search in ten databases, the inclusion of RCTs, the methodological quality assessment and conduction of a meta-analysis are strengths of the study. Further, by only including studies focusing on yoga interventions that contained physical postures (asanas), we attempted to reduce the variability between the yoga interventions, thereby increasing the comparability of studies. Nevertheless, there may still remain some variability between the different types of yoga interventions included in this review. This may be reflected by the high heterogeneity. Other sources of high heterogeneity may be differences in instruments used to define the outcome, differences in patient groups (i.e. different stage of cancer, or different timing of the intervention with respect to primary cancer treatment), or differences in control groups. Because of the small number of studies, we were unable to conduct subgroup analyses to further reduce heterogeneity. Although we used random effects modelling to take into account the large heterogeneity, overall effect sizes should be interpreted with caution as they may vary somewhat among subgroups.

In general, publication bias endangers the external validity of reviews and meta-analyses. Also in this study, publication bias cannot be ruled out. Another limitation is the small sample size of some studies. In addition, some studies were conducted by the same research group, and other studies had multiple outcomes, increasing the probability of type 1 errors. Furthermore, most studies offered yoga to people based on having cancer, not based on having physical or psychosocial problems, which may have resulted in an underestimation of the beneficial effects of yoga.

The yoga interventions were conducted during various forms of cancer treatment; some were conducted posttreatment, and some studies included a mixed sample of patients during and post-treatment. Due to the limited number of studies, it is difficult to draw conclusions on the optimal timing for yoga interventions. Future studies should consider this issue.

Further, although effect sizes for many psychosocial outcomes were generally moderate to large, effect sizes of yoga interventions on physical function and functional well-being were small. This may indicate that the effect is small, or that some patients may have physical benefits from yoga whereas others may not, which may be indicative of the heterogeneity of cancer patients. Future studies with large sample sizes should identify moderators of the effect of yoga on physical and psychosocial outcomes in order to identify subgroups of patients whom may specifically benefit from yoga.

Finally, this review only included papers published in the English language. Although our searches were not limited to language, we may have missed important findings from yoga in Asia, in which practicing yoga is much more common than in Western countries. Nevertheless, this review included six studies that were conducted in Asia.

\section{Clinical implications}

The emerging literature provides preliminary support for the feasibility and efficacy of yoga interventions for cancer patients. Only one adverse effect was reported in five studies that assessed adverse events, and the results indicate that yoga may improve physical well-being and psychosocial outcomes. Although the literature suggests yoga may be effective in improving physical outcomes in other patients and healthy elderly [29,30,34], evidence in cancer patients and survivors is generally insufficient to draw firm conclusions at this stage. In contrast, physical exercise has been shown to be effective in improving physical function and fitness in cancer patients and survivors $[3,4,10,16]$. However, for cancer patients and survivors who are unable or unwilling to participate in traditional aerobic or resistance exercise programs yoga may be an appropriate form of exercise [63] as it is especially suitable for those who perceive barriers to other forms of exercise [69]. Breast cancer patients have been found to perceive more barriers to exercise than age-matched controls, and higher barriers were associated with less exercise [70]. In a recent pilot study, breast cancer survivors reported minimal barriers and high motivation for participating in a yoga program [63]. All participants reported that yoga was beneficial and enjoyable, that they were confident that they could do the exercises, and that they were motivated to attend all classes [63]. However, evidence for yoga as an effective intervention to improve physical function and fitness is lacking and should be established by future studies. Future studies should also systematically assess and report adverse events related to yoga.

\section{Conclusion}

This systematic review and meta-analysis of RCTs showed that yoga has strong beneficial effects on distress, anxiety and depression, moderate effects on fatigue, general HRQoL, emotional function and social function, small effects on functional well-being, and no significant effects on physical function and sleep disturbances. Results of the current review must be interpreted with caution due to the relative small sample sizes of most of the included studies. RCTs with larger sample sizes are needed to improve our understanding of the physical and psychosocial effects of yoga. Future studies should also address the optimal duration and frequency of yoga, the effects in patients with types of cancer other than breast cancer, and the optimal time point in the cancer and cancer treatment or rehabilitation trajectories for offering yoga interventions [63]. 


\section{Competing interests}

The authors declare that they have no competing interests.

\section{Authors' contributions}

$L B, J V U$ and MC have made substantial contributions to conception and design of the manuscript. $L B$ and JVU have screened papers and conducted the quality rating and meta-analysis. IR has conducted the literature search. $L B$ and JVU have been involved in drafting the manuscript. MC, IR, WB, JB, WvM have been involved in critically revising the manuscript. All authors read and approved the final manuscript.

\section{Acknowledgments}

The contribution of LM Buffart was supported by a fellowship granted by the EMGO Institute for Health and Care Research and a grant from the Alpe d'HuZes/KWF Fund, provided by the Dutch Cancer Society. JGZ van Uffelen was supported by a NHMRC program grant (Owen, Bauman and Brown; \#569663) at The University of Queensland, School of Human Movement Studies.

\section{Author details}

${ }^{1}$ EMGO Institute for Health and Care Research, Department of Epidemiology and Biostatistics, VU University Medical Center, Van der Boechorststraat 7, Amsterdam 1081 BT, The Netherlands. ${ }^{2}$ Institute of Sport, Exercise and Active Living, Victoria University, Victoria, Australia. ${ }^{3}$ School of Human Movement Studies, The University of Queensland, Queensland, Australia. ${ }^{4}$ Faculty of Medicine, Norwegian University of Science and Technology, Trondheim, Norway. ${ }^{5}$ EMGO Institute for Health and Care Research, Department of Public and Occupational Health, VU University Medical Center, Amsterdam, The Netherlands.

Received: 15 February 2012 Accepted: 21 November 2012 Published: 27 November 2012

\section{References}

1. Australian Institute of Health and Welfare: Cancer survival and prevalence in Australia. Canberra: CAT no CAN 38; 2008.

2. IKCnet.Dutch Cancer Registration: Survival. 2009. http://www.ikcnet.nl.

3. Courneya KS, Friedenreich CM: Physical exercise and quality of life following cancer diagnosis: a literature review. Ann Behav Med 1999 21:171-179.

4. Courneya KS: Exercise in cancer survivors: an overview of research. Med Sci Sports Exerc 2003, 35:1846-1852.

5. Curt GA, Breitbart W, Cella D, Groopman JE, Horning SJ, Itri LM, et al: Impact of cancer-related fatigue on the lives of patients: new findings from the Fatigue Coalition. Oncologist 2000, 5:353-360.

6. Dimeo FC: Effects of exercise on cancer-related fatigue. Cancer 2001, 92:1689-1693.

7. Emslie C, Whyte F, Campbell A, Mutrie N, Lee L, Ritchie D, et al: 'I wouldn't have been interested in just sitting round a table talking about cancer'; exploring the experiences of women with breast cancer in a group exercise trial. Health Educ Res 2007, 22:827-838.

8. Cheema B, Gaul CA, Lane K, Fiatarone Singh MA: Progressive resistance training in breast cancer: a systematic review of clinical trials. Breast Cancer Res Treat 2008, 109:9-26.

9. Conn CA, Kozak WE, Tooten PC, Gruys E, Borer KT, Kluger MJ: Effect of voluntary exercise and food restriction in response to lipopolysaccharide in hamsters. J Appl Physiol 1995, 78:466-477.

10. De Backer IC, Schep G, Backx FJ, Vreugdenhil G, Kuipers H: Resistance training in cancer survivors: a systematic review. Int J Sports Med 2009 30:703-712.

11. Galvao DA, Newton RU: Review of exercise intervention studies in cancer patients. J Clin Oncol 2005, 23:899-909.

12. Jacobsen PB, Donovan KA, Vadaparampil ST, Small BJ: Systematic review and meta-analysis of psychological and activity-based interventions for cancer-related fatigue. Health Psychol 2007, 26:660-667.

13. Kangas M, Bovbjerg DH, Montgomery GH: Cancer-related fatigue: a systematic and meta-analytic review of non-pharmacological therapies for cancer patients. Psychol Bull 2008, 134:700-741.

14. Kirshbaum MN: A review of the benefits of whole body exercise during and after treatment for breast cancer. J Clin Nurs 2007, 16:104-121.
15. Knols R, Aaronson NK, Uebelhart D, Fransen J, Aufdemkampe G: Physical exercise in cancer patients during and after medical treatment: a systematic review of randomized and controlled clinical trials. J Clin Oncol 2005, 23:3830-3842.

16. Speck RM, Courneya KS, Masse LC, Duval S, Schmitz KH: An update of controlled physical activity trials in cancer survivors: a systematic review and meta-analysis. J Cancer Surviv 2010, 4:87-100.

17. Courneya KS, McKenzie DC, Reid RD, Mackey JR, Gelmon K, Friedenreich CM, et al: Barriers to supervised exercise training in a randomized controlled trial of breast cancer patients receiving chemotherapy. Ann Behav Med 2008, 35:116-122.

18. Midtgaard J, Baadsgaard MT, Moller T, Rasmussen B, Quist M, Andersen C, et al: Self-reported physical activity behaviour; exercise motivation and information among Danish adult cancer patients undergoing chemotherapy. Eur J Oncol Nurs 2009, 13:116-121.

19. Rogers LQ, Courneya KS, Verhulst S, Markwell SJ, McAuley E: Factors associated with exercise counseling and program preferences among breast cancer survivors. J Phys Act Health 2008, 5:688-705.

20. Whitehead S, Lavelle K: Older breast cancer survivors' views and preferences for physical activity. Qual Health Res 2009, 19:894-906.

21. Courneya KS, Friedenreich CM, Quinney HA, Fields AL, Jones LW, Vallance $\mathrm{JK}$, et al: A longitudinal study of exercise barriers in colorectal cancer survivors participating in a randomized controlled trial. Ann Behav Med 2005, 29:147-153.

22. Perna FM, Craft L, Carver CS, Antoni MH: Negative affect and barriers to exercise among early stage breast cancer patients. Health Psychol 2008, 27:275-279.

23. Courneya KS, Friedenreich CM, Quinney HA, Fields AL, Jones LW, Fairey AS: Predictors of adherence and contamination in a randomized trial of exercise in colorectal cancer survivors. Psychooncology 2004, 13:857-866.

24. Ernst E, Cassileth BR: The prevalence of complementary/alternative medicine in cancer: a systematic review. Cancer 1998, 83:777-782.

25. Bernstein BJ, Grasso T: Prevalence of complementary and alternative medicine use in cancer patients. Oncology (Williston Park) 2001, 15:1267-1272.

26. Molassiotis A, Fernadez-Ortega P, Pud D, Ozden G, Scott JA, Panteli V, et al: Use of complementary and alternative medicine in cancer patients: a European survey. Ann Oncol 2005, 16:655-663.

27. Lipton $L$ : Using yoga to treat disease: an evidence-based review. JAAPA 2008, 21:34-36. 38, 41.

28. Telles S, Ramaprabhu V, Reddy SK: Effect of yoga training on maze learning. Indian J Physiol Pharmacol 2000, 44:197-201.

29. Raub JA: Psychophysiologic effects of Hatha Yoga on musculoskeletal and cardiopulmonary function: a literature review. J Altern Complement Med 2002, 8:797-812.

30. Tran MD, Holly RG, Lashbrook J, Amsterdam EA: Effects of Hatha Yoga Practice on the Health-Related Aspects of Physical Fitness. Prev Cardiol 2001, 4:165-170.

31. Blank SE, Kittel J, Haberman MR: Active practice of lyengar Yoga as an intervention for breast cancer survivors. Int J Yoga Therapy 2010, 15:51-59.

32. Carson JW, Carson KM, Porter LS, Keefe FJ, Seewaldt VL: Yoga of Awareness program for menopausal symptoms in breast cancer survivors: results from a randomized trial. Support Care Cancer 2009, 17:1301-1309.

33. Galantino M, Cannon N, Hoelker T, lannaco J, Quinn L: Potential benefits of walking and yoga on perceived level of cognitive decline and persistent fatigue in women with breast cancer. Rehabil Oncol 2007, 25:3-16.

34. Ross A, Thomas S: The health benefits of yoga and exercise: a review of comparison studies. J Altern Complement Med 2010, 16:3-12.

35. Smith KB, Pukall CF: An evidence-based review of yoga as a complementary intervention for patients with cancer. Psychooncology 2009, 18:465-475.

36. Bower JE, Woolery A, Sternlieb B, Garet D: Yoga for cancer patients and survivors. Cancer Control 2005, 12:165-171.

37. Lin KY, Hu YT, Chang KJ, Lin HF, Tsauo JY: Effects of yoga on psychological health, quality of life, and physical health of patients with cancer: a meta-analysis. Evid Based Complement Alternat Med 2011, Epub.

38. Verhagen AP, de Vet HC, de Bie RA, Boers M, van den Brandt PA: The art of quality assessment of RCTs included in systematic reviews. J Clin Epidemiol 2001, 54:651-654.

39. Chinapaw MJ, van Uffelen JG, Riphagen I, van Mechelen W: The functional effects of physical exercise training in frail older people: a systematic review. Sports Med 2008, 38:781-793. 
40. van Uffelen JG, Chinapaw MJ, Hopman-Rock M, van Mechelen W: The effects of exercise on cognition in older adults with and without cognitive decline: a systematic review. Clin J Sport Med 2008, 18:486-500.

41. Verhagen AP, Karels C, Bierma-Zeinstra SM, Feleus A, Dahaghin S, Burdorf A, et al: Exercise proves effective in a systematic review of work-related complaints of the arm, neck, or shoulder. J Clin Epidemiol 2007, 60:110-117.

42. Cuijpers $P$, van Straten A, Bohlmeijer E, Hollon SD, Andersson G: The effects of psychotherapy for adult depression are overestimated: a metaanalysis of study quality and effect size. Psychol Med 2010, 40:211-223.

43. Lipsey MW: Design sensitivity; statistical power for experimental research. Newbury Park: Sage; 1990.

44. Cohen L, Warneke C, Fouladi RT, Rodriguez MA, Chaoul-Reich A: Psychological adjustment and sleep quality in a randomized trial of the effects of a Tibetan yoga intervention in patients with lymphoma. Cancer 2004, 100:2253-2260.

45. Higgins JP, Thompson SG, Deeks JJ, Altman DG: Measuring inconsistency in meta-analyses. BMJ 2003, 327:557-560

46. Vadiraja HS, Raghavendra RM, Nagarathna R, Nagendra HR, Rekha M, Vanitha $N$, et al: Effects of a yoga program on cortisol rhythm and mood states in early breast cancer patients undergoing adjuvant radiotherapy: a randomized controlled trial. Integr Cancer Ther 2009, 8:37-46.

47. Vadiraja HS, Rao MR, Nagarathna R, Nagendra HR, Rekha M, Vanitha N, et al: Effects of yoga program on quality of life and affect in early breast cancer patients undergoing adjuvant radiotherapy: a randomized controlled trial. Complement Ther Med 2009, 17:274-280.

48. Vadiraja HS, Rao RM, Hongasandra NR, Nagarathna R, Rekha M, Vanitha N, et al: Effects of yoga on symptom management in breast cancer patients: A randomized controlled trial. Int J Yoga 2009, 2:73-79.

49. Raghavendra RM, Nagarathna R, Nagendra HR, Gopinath KS, Srinath BS, Ravi $B D$, et al: Effects of an integrated yoga programme on chemotherapyinduced nausea and emesis in breast cancer patients. Eur J Cancer Care (Engl) 2007, 16:462-474.

50. Rao RM, Raghuram N, Nagendra HR, Gopinath KS, Srinath BS, Diwakar RB, et al: Anxiolytic effects of a yoga program in early breast cancer patients undergoing conventional treatment: a randomized controlled trial. Complement Ther Med 2009, 17:1-8.

51. Banerjee B, Vadiraj HS, Ram A, Rao R, Jayapal M, Gopinath KS, et al: Effects of an integrated yoga program in modulating psychological stress and radiation-induced genotoxic stress in breast cancer patients undergoing radiotherapy. Integr Cancer Ther 2007, 6:242-250.

52. Culos-Reed SN, Carlson LE, Daroux LM, Hately-Aldous S: A pilot study of yoga for breast cancer survivors: physical and psychological benefits. Psychooncology 2006, 15:891-897.

53. Danhauer SC, Mihalko SL, Russell GB, Campbell CR, Felder L, Daley K, et al: Restorative yoga for women with breast cancer: findings from a randomized pilot study. Psychooncology 2009, 18:360-368.

54. Moadel AB, Shah C, Wylie-Rosett J, Harris MS, Patel SR, Hall CB, et al: Randomized controlled trial of yoga among a multiethnic sample of breast cancer patients: effects on quality of life. J Clin Oncol 2007, 25:4387-4395

55. Chandwani KD, Thornton B, Perkins GH, Arun B, Raghuram NV, Nagendra $H R$, et al: Yoga improves quality of life and benefit finding in women undergoing radiotherapy for breast cancer. J Soc Integr Oncol 2010, 8:43-55.

56. Banasik J, Williams H, Haberman M, Blank SE, Bendel R: Effect of lyengar yoga practice on fatigue and diurnal salivary cortisol concentration in breast cancer survivors. J Am Acad Nurse Pract 2011, 3:135-142.

57. Littman AJ, Bertram LC, Ceballos R, Ulrich CM, Ramaprasad J, McGregor B, et al: Randomized controlled pilot trial of yoga in overweight and obese breast cancer survivors: effects on quality of life and anthropometric measures. Support Care Cancer 2012, 20:267-277.

58. Bower JE, Garet D, Sternlieb B, Ganz PA, Irwin MR, Olmstead R, et al: Yoga for persistent fatigue in breast cancer survivors: A randomized controlled trial. Cancer 2012, 118:3766-3775.

59. Ainsworth BE, Haskell WL, Leon AS, Jacobs DR Jr, Montoye HJ, Sallis JF, et al: Compendium of physical activities: classification of energy costs of human physical activities. Med Sci Sports Exerc 1993, 25:71-80.

60. Oken BS, Zajdel D, Kishiyama S, Flegal K, Dehen C, Haas M, et al: Randomized, controlled, six-month trial of yoga in healthy seniors: effects on cognition and quality of life. Altern Ther Health Med 2006, 12:40-47.
61. Pullen PR, Nagamia SH, Mehta PK, Thompson WR, Benardot D, Hammoud R, et al: Effects of yoga on inflammation and exercise capacity in patients with chronic heart failure. J Card Fail 2008, 14:407-413.

62. Bowman AJ, Clayton RH, Murray A, Reed JW, Subhan MM, Ford GA: Effects of aerobic exercise training and yoga on the baroreflex in healthy elderly persons. Eur J Clin Invest 1997, 27:443-449.

63. Speed-Andrews AE, Stevinson C, Belanger LJ, Mirus JJ, Courneya KS: Pilot evaluation of an iyengar yoga program for breast cancer survivors. Cancer Nurs 2010, 33:369-381.

64. de Jong N: Courtens AM, bu-Saad HH, Schouten HC: Fatigue in patients with breast cancer receiving adjuvant chemotherapy: a review of the literature. Cancer Nurs 2002, 25:283-297.

65. Cramp F, Daniel J: Exercise for the management of cancer-related fatigue in adults. Cochrane Database Syst Rev 2008, (2):CD006145.

66. Duijts SF, Faber MM, van Oldenburg HS BM, Aaronson NK: Effectiveness of behavioral techniques and physical exercise on psychosocial functioning and health-related quality of life in breast cancer patients and survivors-a meta-analysis. Psychooncology 2011, 20:115-126.

67. Velthuis MJ, Agasi-Idenburg SC, Aufdemkampe G, Wittink HM: The effect of physical exercise on cancer-related fatigue during cancer treatment: a meta-analysis of randomised controlled trials. Clin Oncol (R Coll Radiol) 2010, 22:208-221.

68. Goedendorp MM, Gielissen MF, Verhagen CA, Bleijenberg G: Psychosocial interventions for reducing fatigue during cancer treatment in adults. Cochrane Database Syst Rev 2009, (1):CD006953.

69. Brawley LR, Culos-Reed SN, Angove J, Hoffman-Goetz L: Understanding the barriers to phyiscal activity for cancer patients: review and recommendations. J Psychosoc Oncol 2002, 20:1-21.

70. Courneya KS, Friedenreich CM: Relationship between exercise pattern across the cancer experience and current quality of life in colorectal cancer survivors. J Altern Complement Med 1997, 3:215-226.

doi:10.1186/1471-2407-12-559

Cite this article as: Buffart et al:: Physical and psychosocial benefits of yoga in cancer patients and survivors, a systematic review and metaanalysis of randomized controlled trials. BMC Cancer 2012 12:559.

\section{Submit your next manuscript to BioMed Central and take full advantage of:}

- Convenient online submission

- Thorough peer review

- No space constraints or color figure charges

- Immediate publication on acceptance

- Inclusion in PubMed, CAS, Scopus and Google Scholar

- Research which is freely available for redistribution

Submit your manuscript at www.biomedcentral.com/submit
C) Biomed Central 